\title{
The circumstellar environment of the YSO TMR-1 and a revisit to the candidate very low-mass object TMR-1C ${ }^{\star}$
}

\author{
M. G. Petr-Gotzens ${ }^{1}$, J.-G. Cuby², M. D. Smith ${ }^{3}$, and M. F. Sterzik ${ }^{4}$ \\ ${ }^{1}$ European Southern Observatory, Karl-Schwarzschild-Str. 2, 85748 Garching, Germany \\ e-mail: mpetr@eso.org \\ ${ }^{2}$ Laboratoire d'Astrophysique de Marseille, OAMP, Université Aix-Marseille \& CNRS, 38 rue Frédéric Joliot Curie, \\ 13388 Marseille Cedex 13, France \\ 3 Centre for Astrophysics and Planetary Science, University of Kent, Canterbury, Kent CT2 7NR, UK \\ 4 European Southern Observatory, Casilla 19001, Santiago 19, Chile
}

Received 26 May 2009 / Accepted 27 July 2010

\begin{abstract}
Context. TMR-1 (IRAS 04361+2547) is a class I proto-stellar source located in the nearby Taurus star-forming region. Its circumstellar environment is characterized by extended dust emission with complex structures and conspicuous filaments. A faint companion, called TMR-1C, located near the proto-star was detected in previous studies, but its nature as a very young substellar object remains inconclusive.

Aims. We aim to improve the constraints on the nature of the faint object TMR-1C, and to investigate the process of very low-mass star formation in the TMR-1 system.

Methods. Using very sensitive infrared imaging observations of the TMR-1 system and near-infrared spectroscopy, we compile the spectral energy distribution (SED) of TMR-1C over a much wider wavelength range than possible in previous work. We then compare the spectral energy distribution with models of extincted background stars, young sub-stellar objects, and very low-mass stars with circumstellar disk and envelope emission. We also search for additional low-luminosity sources in the immediate environment of the TMR-1 proto-stellar source. Furthermore, we study the surrounding near-infrared dust morphology, and analyse the emission line spectrum of a filamentary structure in the physical context of a bow-shock model.

Results. We find that the observed SED of TMR-1C can be reproduced by neither an extincted background star, nor available models for a young extremely low-mass $\left(\lesssim 12 M_{\mathrm{J}}\right)$ object. Our near-infrared spectrum allows us to infer an effective temperature $\gtrsim 3000 \mathrm{~K}$. We achieve a close fit of TMR-1C's SED using radiation transfer models of young stellar objects with circumstellar disks, hence propose that TMR-1C is most likely a very low-mass star with $M \approx 0.1-0.2 M_{\odot}$ surrounded by a circumstellar disk of high inclination, $i>80^{\circ}$. Interestingly, we detect an additional very faint source, which we call TMR-1D, that is remarkably symmetrical in terms of position with TMR-1C. Both TMR-1C and TMR-1D may have formed from a common star-formation event, triggered by a powerful outflow or by the collision of primordial proto-stellar disks. The impact of an outflow is traced by molecular hydrogen emission that we detect from a distinct filament pointing towards TMR-1C. A comparison with C-type bow shock models confirms that the emission is caused by shock excitation.
\end{abstract}

Key words. stars: formation - stars: pre-main sequence - circumstellar matter - stars: low-mass stars: individual: IRAS $04361+2547$ - Shock waves

\section{Introduction}

TMR-1 (IRAS 04361+2547) is a class I young stellar object located in the Taurus molecular cloud, which was resolved into a binary source with a measured components' separation of $0.31^{\prime \prime}$ (Terebey et al. 1998, hereafter T98). We therefore refer to it as TMR-1AB in the following. The total bolometric luminosity of TMR-1AB was estimated to be $\sim 2.8 L_{\odot}$, indicative of a low-mass protostellar system (Kenyon et al. 1993). The circumstellar environment of TMR-1AB is characterized by extended emission from both a dusty proto-stellar envelope and patches of molecular cloud material remaining from the protostellar collapse. In their sensitive HST/NICMOS observations, T98 detected a faint compact object, named TMR-1C, at a projected distance of $\sim 10^{\prime \prime}$ from TMR-1AB, which corresponds to

* Based on observations made with ESO telescopes at the La Silla Paranal Observatory under programme ID 265.C-5747(A), and based on data obtained from the ESO Science Archive Facility. $\sim 1400 \mathrm{AU}$ at the distance of the Taurus molecular cloud. The physical association of TMR-1C with TMR-1AB was implied by a striking filament structure originating in TMR-1 that points directly towards TMR-1C. T98 suggested that TMR1-C was catapulted to its current location by dynamical interactions with the proto-binary TMR1-AB and that the arc-shaped filament could trace the ejection path of TMR-1C through the gaseous infalling circumstellar envelope of TMR-1. The very low luminosity suggested for TMR-1C indicated that it should be a substellar object, maybe even a planetary mass object. However, the physical association of TMR-1C with TMR-1, hence its nature as a substellar object, was strongly debated during the years after its discovery. In an attempt to clarify the evolutionary status of TMR-1C, Terebey et al. (2000) carried out near-infrared spectroscopy using the Keck telescope. The result of these observations showed that the spectrum of TMR-1C, at the signalto-noise ratio (hereafter $S / N$ ) level that could be reached, is consistent with that of an extincted background dwarf star, but still 
Table 1. Infrared photometry of TMR-1C.

\begin{tabular}{clcc}
\hline \hline Filter & $\begin{array}{l}\text { Central } \\
\text { Wavelength } \\
(\mu \mathrm{m})\end{array}$ & $\begin{array}{c}\text { Magnitude } \\
\text { or Flux } \\
(\mathrm{mag} \text { or Jy) }\end{array}$ & $\begin{array}{c}\text { Date of observation } \\
(\mathrm{UT})\end{array}$ \\
\hline$H$ & 1.65 & $19.21 \pm 0.05$ & Oct. 01 + 02, 2000 \\
$K_{\mathrm{s}}$ & 2.16 & $17.63 \pm 0.1$ & Dec. 05, 1998 \\
$K_{\mathrm{s}}$ & 2.16 & $17.53 \pm 0.05$ & Oct. 01, 2000 \\
$L^{\prime}$ & 3.78 & $<7.5 \times 10^{-5}$ & Sept. 20 + Oct. 02, 2000 \\
IRAC CH1 & 3.6 & $<0.12 \times 10^{-3}$ & Mar. 07, 2004 \\
IRAC CH2 & 4.5 & $<0.51 \times 10^{-3}$ & Mar. 07, 2004 \\
\hline
\end{tabular}

room was left for an interpretation within the extremely lowmass object ejection hypothesis.

In this paper, we use ESO Very Large Telescope (VLT) data obtained with the Infrared Spectrometer and Array Camera (ISAAC), as well as Spitzer/IRAC observations to revisit the TMR-1 system. After the description of the observations and the data reduction (Sect. 2), we discuss in Sect. 3.1 the morphology of the circumstellar dusty environment of TMR-1 and report the detection of new features and objects identified in our sensitive ISAAC images. We then use our $K$-band low-resolution spectroscopy together with the spectral energy distribution of TMR-1C, which we compile from the photometry presented in this paper and collected from the literature, to analyze the nature of TMR-1C in Sects. 3.2 and 3.3. Our $K$-band spectroscopy was also performed on a significant part of the filament structure "connecting" TMR-1C with TMR-1AB. Since the spectral resolution is almost 4 times higher than in previous spectroscopic observations, we are able to resolve numerous emission-line features arising from the filament. In Sect. 3.4, we analyze the filament spectrum in detail, which leads us to conclude that a significant part of its emission is characterized by shock-excited emission. In Sect. 4, we present scenarios that could possibly explain the symmetry of the circumstellar nebulosities, the filamentary structures, and the existence of a pair of very low-mass objects, as a physical entity. Finally, we summarize our results and conclusions in Sect. 5.

\section{Imaging and spectroscopy data}

\subsection{Near-infrared photometry from VLT/ISAAC HK observations}

A first set of images of TMR-1 was obtained at $K$ s-band $\left(\lambda_{\mathrm{c}}=\right.$ $2.16 \mu \mathrm{m}, \Delta \lambda=0.27 \mu \mathrm{m}$ ) with ISAAC at the VLT-ANTU(UT1) telescope as part of the commissioning of the instrument. These data were taken during the night December 04-05, 1998, immediately before the spectroscopic observations, providing also a flux calibration for these data (Sect. 2.3). The data are accessible via the ESO Science Archive Facility ${ }^{1}$. The scale of the ISAAC/Hawaii Rockwell detector was $0.147^{\prime \prime} /$ pix, yielding a field-of-view of $2.5^{\prime} \times 2.5^{\prime}$. The images typically consisted of 50 frames averaged with $1.77 \mathrm{~s}$ individual exposure time. Those having the best image quality, of the order of $\sim 0.55^{\prime \prime} F W H M$, were selected for further data reduction. The sky contribution was determined from median combining the individual images, and then subtracted from each image. Finally, the sky subtracted images were bad-pixel corrected, aligned and combined, and trimmed to a common overlap region. The total integration time in the final mosaicked image was $177 \mathrm{~s}$.

\footnotetext{
${ }^{1}$ http://archive.eso.org/eso/eso_archive_main.html
}

A second set of ISAAC near-infrared data was obtained at $H$-band $\left(\lambda_{\mathrm{c}}=1.65 \mu \mathrm{m}, \Delta \lambda=0.30 \mu \mathrm{m}\right)$, and again at $K_{\mathrm{S}}$-band, on two nights during October 2000 (Table 1). The images were taken by using a random jitter pattern centered on TMR-1. To avoid strong saturation from the brightest source, TMR-1 AB, short detector integration times of DIT $=2 \mathrm{~s}$ and DIT $=3 \mathrm{~s}$ were used for $K_{\mathrm{S}}$ and $H$ exposures, respectively. The total exposure times per image were $2 \times 20$ (DIT $\times$ NDIT) sec for $K_{\mathrm{S}}$ images and $3 \times 20(\mathrm{DIT} \times \mathrm{NDIT}) \mathrm{sec}$ for $\mathrm{H}$ images. The data were then processed with the Eclipse ${ }^{2}$ data reduction package (Devillard 1997), using the jitter algorithm. The images were dark-subtracted and flat-fielded with a skyflat obtained from exposures taken during twilight, then sky-subtracted, and finally recentered and stacked to produce single deep $H$ - and $K$ s-band images. The total integration time in the stacked $\mathrm{H}$-image is $12 \mathrm{~min}$, while it is $20 \mathrm{~min}$ for the Ks-image. TMR-1 and its immediate surrounding as seen in these sensitive images is shown in Figs. 1a-c. The measured image qualities are very good, with $F W H M \sim 0.5^{\prime \prime}(K \mathrm{~s})$ and $F W H M \sim 0.7^{\prime \prime}(\mathrm{H})$, but still not good enough to resolve the binary TMR-1AB.

Accurate photometry for all sources with a minimum $S / N$ detection of 8 was then performed on the $H$ and $K$ s mosaic images by using PSF-fitting with IRAF/daophot. In particular, for TMR-1C, which is close to the nebulous filament and the general nebulosity surrounding TMR1, PSF photometry provides a far more accurate photometric measurement than aperture photometry. In all of our final $K$ s and $H$ mosaics, TMR- $1 C$ has been well detected at a $S / N$ of $\geq 15$. For the observations carried out in 2000 , the absolute photometric calibration has been derived from the UKIRT faint standard FS12 that was observed immediately after our observations of TMR-1. The resulting photometry for TMR-1C is $K \mathrm{~s}=17.53 \mathrm{mag}$ and $H=19.21 \mathrm{mag}$ with an uncertainty of $\sim 5 \%$. We checked the photometric calibration against the photometry of a 2 MASS star that is present in the ISAAC field (2MASS 04391199+2553490), and find agreement within 0.06 mag. Our $K$ s photometry of TMR-1C is also consistent with values reported by other authors at similar wavelengths (Itoh et al. 1999), and also in agreement with the Ks-photometry determined from the 1998 imaging data (see Table 1). The photometric accuracy for the 1998 data is worse due to the lack of standard star observations during the observing night, so that absolute photometry was derived assuming the $K$ s-zeropoint determined for the ISAAC commissioning I period.

Our deep imaging photometry revealed numerous faint objects, detected all over the field-of-view (Fig. 1a). There are more than 20 objects with a $K$ s-magnitude similar or fainter than TMR-1C, indicating that we are beginning to penetrate through the large amount of dust in the molecular cloud core. In this respect, the faintness and appearance of TMR-1C seems not to be

\footnotetext{
$\overline{2}$ http://www.eso.org/projects/aot/eclipse/
} 

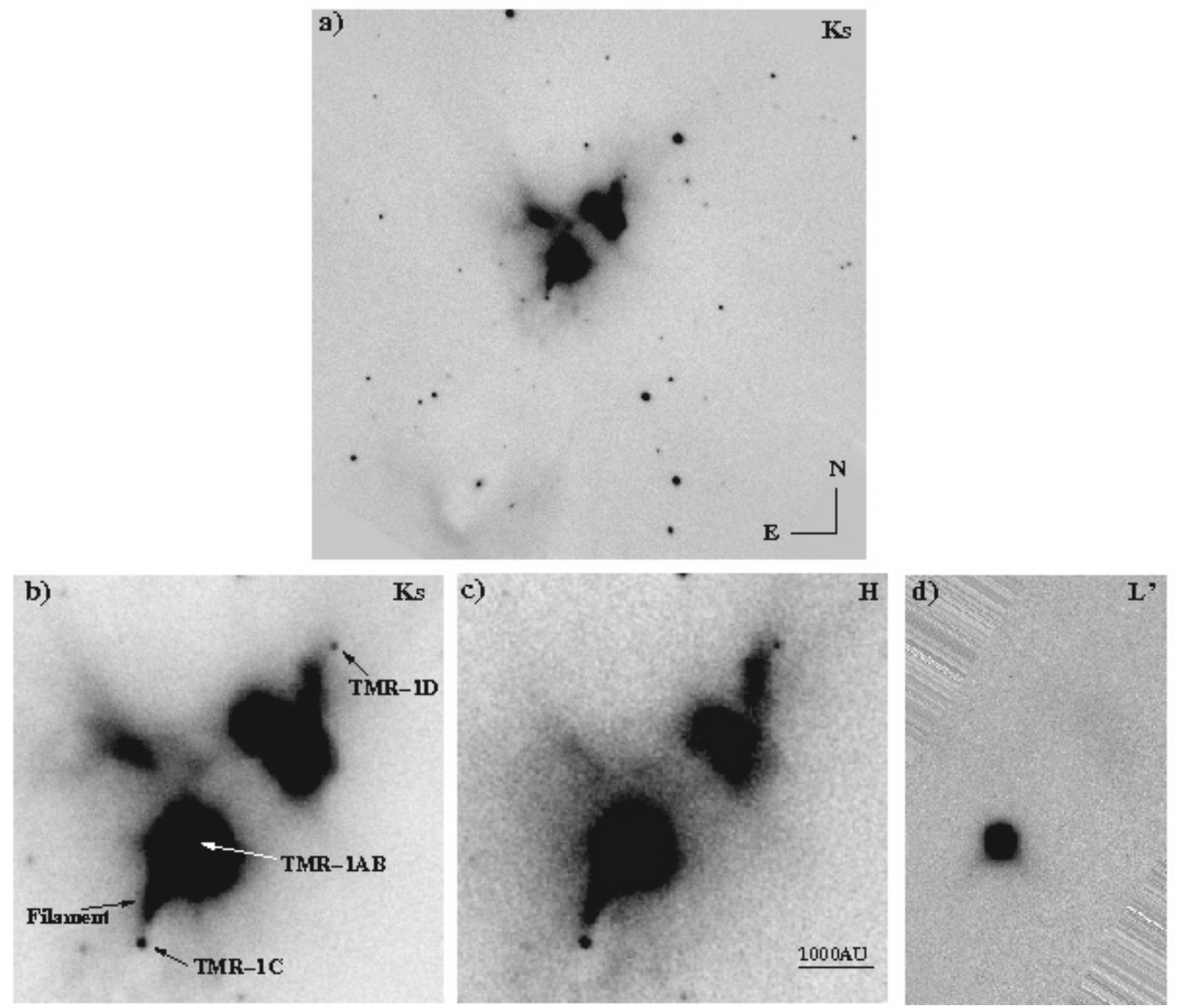

Fig. 1. a) ISAAC Ks $(2.16 \mu \mathrm{m})$ image of TMR-1 and its surroundings. The total integration time in this image is 20 min and the image size is approximately $2.4^{\prime} \times 2.4^{\prime}$; b) zoom-in on the Ks-image of TMR-1 clearly showing the candidate very low-mass object TMR-1C at the end of the filament structure arising from the nebulosity around the binary TMR-1AB, and our detection of a "counter-object", TMR-1D. Image size is approximately $40^{\prime \prime} \times 40^{\prime \prime}$; c) $H$-band image of the same field as shown in b); d) $L^{\prime}$-band image of TMR-1, at the same scale and orientation as the fields shown in b) and $\mathbf{c}$ ).

any special, except for its location at the end of the filament. Very interestingly, we also detected a previously unknown, faint object that resembles a TMR-1C counterpart: it is located close to the end of a broad filamentary structure that extends from the large nebulosity seen to the northwest of TMR-1AB (Fig. 1a-c). The object is clearly a $>10 \sigma$ detection in the deep $H$ and $K$ sband images and fainter than TMR-1C by $\sim 1.1 \mathrm{mag}$ at $K \mathrm{~s}$ and by $\sim 1.0 \mathrm{mag}$ at $H$. We refer to this object as TMR-1D hereafter. Although at this point, we do not have a proof that there is any physical relation between this object and TMR-1 or to TMR-1C, we find it quite striking to detect this symmetry. In the course of this paper, we discuss the presence of TMR-1D in the context of different scenarios.

\subsection{Mid-infrared photometry from VLT/ISAAC $L^{\prime}$-band and Spitzer/IRAC observations}

ISAAC mid-infrared imaging at $L^{\prime}$-band $\left(\lambda_{\mathrm{c}}=3.78 \mu \mathrm{m}, \Delta \lambda=\right.$ $0.58 \mu \mathrm{m}$ ), was performed with the long-wavelength imaging camera of ISAAC, equipped with an Aladdin $1024 \times 1024$ pixel array, and providing a pixel scale of $0.071^{\prime \prime} /$ pix in LWI4 mode. Data were taken in uncorrelated/high bias read-out mode using standard chopping and nodding techniques, with a chop-throw position angle of $122^{\circ}$, an amplitude of $30^{\prime \prime}$, and a chopping frequency of $0.1 \mathrm{~Hz}$. The reduction process included bad-pixel filtering and flat-fielding. Positive and negative beams from the $\operatorname{nod} \mathrm{A}$ and B positions were then combined. The total integration time yielded for the final image, shown in Fig. 1d, is 3000 s. TMR-1C is not detected down to the limiting sensitivity level $(3 \sigma)$ of $\sim 7.5 \times 10^{-5} \mathrm{Jy}$. The only source clearly detected in our $L^{\prime}$-band image is indeed TMR-1AB itself. There is also a slight hint of nebulous emission close to TMR-1AB and in the direction of the filament, as well as a faint detection of diffuse emission from the northwestern nebulosity structure. The object TMR-1D was not detected.

We also searched the Spitzer archive for data of TMR-1, and found that observations were obtained as part of the Spitzer/IRAC programmes No. 37 (PI: Fazio) and No. 3584 (PI: Padgett) devoted to IRAC mappings of the Taurus molecular cloud. Since the spatial resolution of IRAC at its 4 wavelengths 

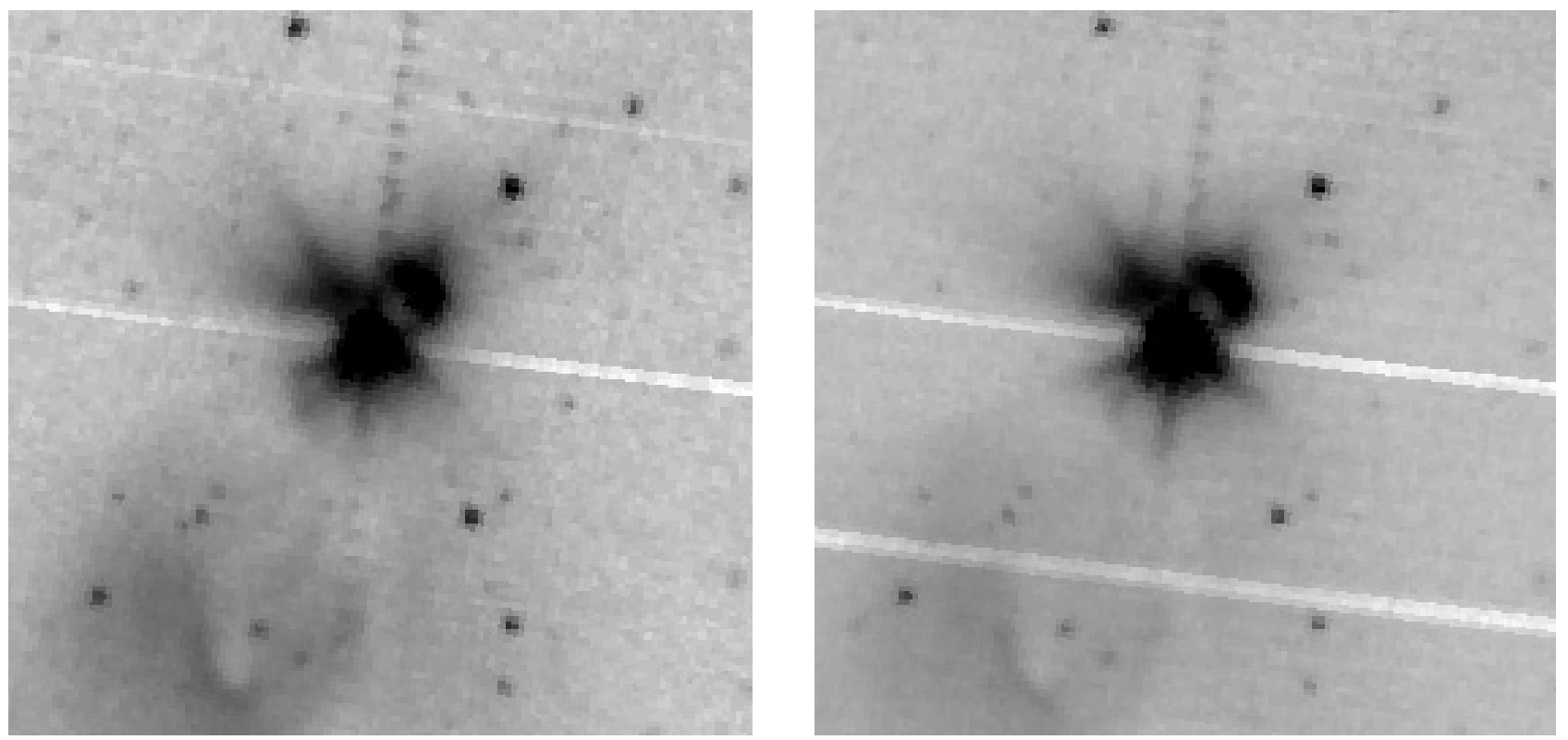

Fig. 2. Spitzer/IRAC image at $3.6 \mu \mathrm{m}$ (left) and $4.5 \mu \mathrm{m}$ (right) showing TMR-1 and its surrounding. North is up and east is to the left, and the image sizes are the same as in Fig. 1a.

channels, ch1 $(3.6 \mu \mathrm{m}), \operatorname{ch} 2(4.5 \mu \mathrm{m}), \operatorname{ch} 3(5.8 \mu \mathrm{m})$, and $\operatorname{ch} 4$ $(8.0 \mu \mathrm{m})$ is on the order of $1^{\prime \prime}$ to $2^{\prime \prime}$ and TMR-1C is almost $10^{\prime \prime}$ away from TMR-1AB, TMR-1C may have been detected if it were bright enough at IRAC wavelengths. Therefore, we retrieved the post-BCD data from the Spitzer archive, which had been reduced with the Spitzer pipeline version S14.0, and investigated the images. The diffuse nebulosities of TMR-1 are well detected in the IRAC $3.6 \mu \mathrm{m}$ and $4.5 \mu \mathrm{m}$ images, which we show in Fig. 2, and they are very similar in appearance to what is seen in our ISAAC $2.2 \mu \mathrm{m}$ image, despite being at much lower spatial resolution. The IRAC channel 1 has a central wavelength close to the ISAAC $L^{\prime}$ filter, but due to its much larger pixels IRAC is more sensitive to extended emission than ISAAC. At IRAC channels 3 and $4(5.8 \mu \mathrm{m}$ and $8.0 \mu \mathrm{m})$, only the main source TMR-1AB is seen. On none of the mosaiced IRAC images TMR-1C is detected. This may partly be due to the closeness of TMR-1C to the very bright main source TMR$1 \mathrm{AB}$, which has a flux of $\sim 110 \mathrm{mJy}$ at $3.6 \mu \mathrm{m}$, and $\sim 145 \mathrm{mJy}$ at $4.5 \mu \mathrm{m}$, but may also be related to an intrinsic faintness of TMR-1C in this wavelength range. Given the local background and the Poisson noise measurement at the expected position of TMR-1C, we estimate upper $3 \sigma$ flux detection limits of $0.12 \mathrm{mJy}$ $(3.6 \mu \mathrm{m})$ and $0.51 \mathrm{mJy}(4.5 \mu \mathrm{m})$. Concerning the $5.8 \mu \mathrm{m}$ and $8.0 \mu \mathrm{m}$ images, the PSF wings of the extremely bright source TMR-1AB prevent any meaningful determination of an upper flux limit. The upper flux limits extracted from the Spitzer data are listed together with the ISAAC photometry in Table 1.

\subsection{VLT/ISAAC $K$-band spectroscopy}

$K$-band low-resolution $(R=450)$ long-slit spectroscopy of TMR-1C, and of the filament was performed with ISAAC during the night Dec. 04-05, 1998. In Fig. 3, we show the slit positioning on TMR-1C and the filament. The width of the slit was $1^{\prime \prime}$ and spectra were obtained by observing the object alternately at two positions along the slit, i.e. by nodding along the slit with a nod-throw size of $40^{\prime \prime}$. An exposure time of $120 \mathrm{~s}$ per individual frame was used and the resulting effective exposure time of the final co-added spectrum is $7680 \mathrm{~s}$. Since the slit was positioned

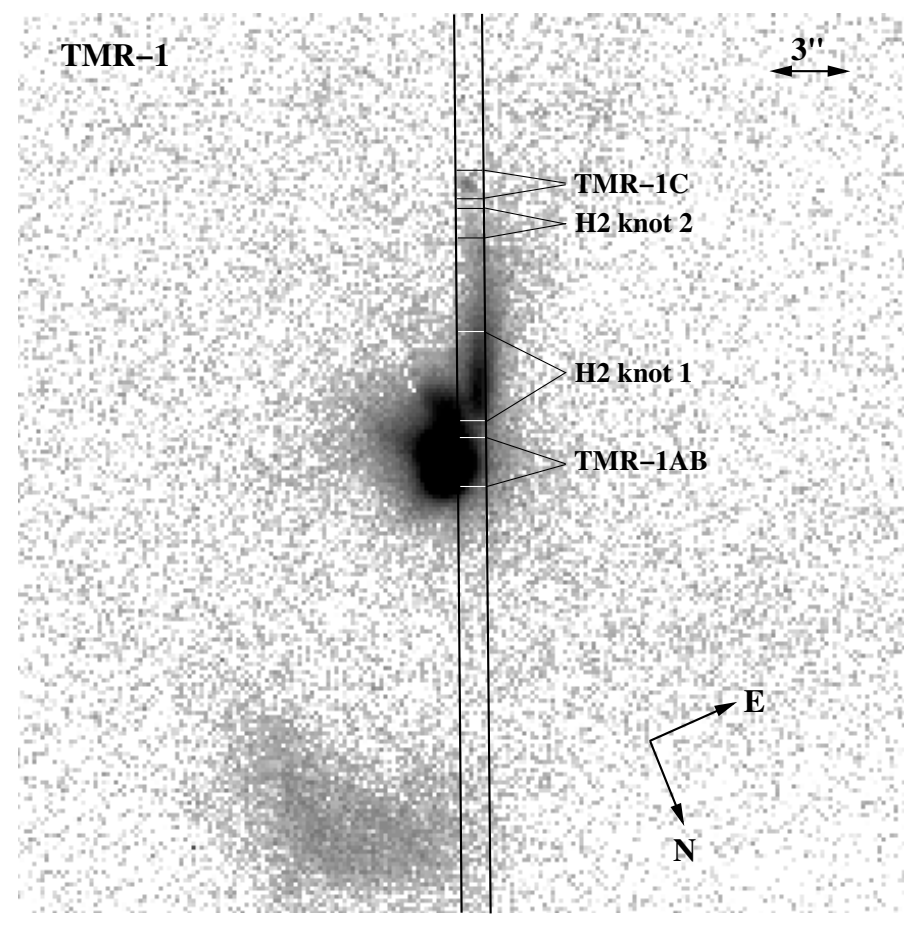

Fig. 3. Position of the slit on TMR-1C and the filament is shown. The sizes and positions of the apertures for the extracted spectra are indicated. The underlying image is the ISAAC $K$ s aquisition image on logarithmic scale.

in a way to include TMR-1C and most of the filament, the binary TMR-1AB was correspondingly slightly out of the slit and only light from its PSF wings and from the immediate circumbinary environment entered the slit.

The spectral images were sky-subtracted in pairs using the respectively nodded companion image for subtraction, cleaned of pixels affected by cosmic rays and non-linear response, and then combined to a single spectral image of TMR-1, which is shown in Cuby et al. (2000). Four different apertures were 
extracted from this 2D-spectrum at positions that are shown in Fig. 3. The largest aperture $\left(\mathrm{H}_{2}\right.$ knot 1$)$ actually includes two separate peaks of molecular hydrogen emission (cf. Fig. 8). Both knots appeared to be very similar on the 2D spectral image and a single aperture was therefore used to extract the spectrum, improving the signal-to-noise ratio.

To correct for telluric absorption, as well as to attempt fluxcalibration, the spectrum of a B6IV standard star (BS3672) was observed directly after TMR-1, with the same instrumental setting and in the same manner (nodding along the slit) as the science spectra. After the basic reduction, the standard star spectrum was divided by a black-body spectrum corresponding to the spectral type of the star from what the absolute spectroscopic response was derived. The extracted TMR-1 spectra were then divided by the spectroscopic response function. Absolute flux calibration based on the spectroscopic observations of the standard star had shown to be difficult, because it relied on the assumption that all flux from the standard star was inside the slit. Moreover, the seeing worsened by a factor of $\sim 1.5$ from the time of observation of TMR-1 to the standard star observation. Consequently, the spectra were calibrated to match the flux density of TMR$1 \mathrm{C}$ as determined from its photometric measurement (Sect. 2.1). Wavelength calibration was made using the $\mathrm{OH}$ sky emission lines and telluric absorption lines. The estimated accuracy in wavelength calibration is on the order of one pixel, i.e. $\sim 7 \AA$. All final four spectra are plotted in Fig. 4.

The following additional uncertainties affect the resulting spectra. Since the standard star spectrum has not been corrected for hydrogen absorption lines, these lines appear in emission in the TMR-1 spectra after division by the response function. In the case of the spectrum close to TMR-1AB and the spectrum $\mathrm{H} 2$ knot 1, however, $\mathrm{Br} \gamma$ emission is also seen in the raw spectrum, while no emission is found in the spectrum $\mathrm{H} 2$ knot 2. Therefore, the Br $\gamma$ line in the first two spectra of Fig. 3 is overestimated. To roughly estimate the true intensity of the $\mathrm{Br} \gamma$ $(2.16 \mu \mathrm{m})$ emission line in these spectra relative to the $2.12 \mu \mathrm{m}$ emission line, the response function was corrected for $\operatorname{Br} \gamma \mathrm{ab}-$ sorption by means of a simple interpolation over the region $\lambda[2.15 \mu \mathrm{m}, 2.175 \mu \mathrm{m}]$. In this way, we estimate that the true intensity ratio of the $\mathrm{Br} \gamma$ to the $2.12 \mu \mathrm{m}$ emission line is $\sim 0.7$ in the spectrum close to TMR-1AB and $\sim 0.14$ and the $\mathrm{H} 2$ knot 1 spectrum. Absorption of the Earth's atmosphere does significantly affect the quality of the spectra at and below $2 \mu \mathrm{m}$, and also to some extent above $2.4 \mu \mathrm{m}$. For instance, below $2 \mu \mathrm{m}$ the spectra are noisier by factors of 4-18 than in the spectral region $\lambda>2 \mu \mathrm{m}$.

Fortunately, a large number of emission lines that we identified in the TMR-1 spectra arise from ro-vibrational transitions of molecular hydrogen, which occupy regions of the spectra that are affected by neither telluric absorption nor the hydrogen absorption lines of the standard star. Therefore the spectral signatures are well measurable. The relative line fluxes of identified emission lines, normalized to the $\mathrm{H}_{2}$ 1-0 S(1) flux, have been measured and are listed in Table 2.

\section{Results and discussion}

\subsection{Morphology of the nebular structure, and new features and detections}

It is well known from previous observations that TMR-1 is associated with bright extended nebulosity consisting of mainly two, a northwestern and a southeastern structure. Our ISAAC images show these structures in great detail (Fig. 1b, c). Each of the two main nebulosities show a filament arm extension, with the filament arm of the northwestern nebulosity being more diffuse than the sharp filament of the southeastern structure. At the end of each filament arm, we detect respectively a point-source, TMR1C and TMR-1D. Another patch of diffuse emission, with an almost rectangular shape, is seen in the Ks-image, located $\sim 10^{\prime \prime}$ north of TMR-1AB. It is significantly less bright in the H-image, suggesting that this structure is more highly extincted.

Considering that TMR-1 is a young stellar object observed during an early evolutionary state, the extended near-infrared emission is interpreted as scattered light from circumstellar dust agglomerations within a dusty proto-stellar envelope and from patches of molecular cloud material remaining from the protostellar collapse. Using near-infrared polarimetric observations, Whitney et al. (1997) report a high degree $(\sim 50 \%)$ of polarized $K$-band emission from both of the main nebulosities and parts of the filaments, indicating that light from the central source TMR-1AB is scattered off from them, and thereby producing most of the emission detected in the $H$ - and $K$-band images. The observations imply that the material of the NW and SE nebulosity must be physically close to TMR-1AB, because otherwise they would not appear in scattered light. A bit surprisingly, the NW nebula appears much fainter at $1.65 \mu \mathrm{m}$ than at $2.16 \mu \mathrm{m}$ and also slightly redder than the SE nebula. We determine $H-K \approx 2.6 \mathrm{mag}$ for the NW nebula and $H-K \approx 2.45 \mathrm{mag}$ for the SE nebula (dominated by the source TMR-1AB). A large clump of dense gas that sits partly in front of the NW nebulosity, detected in the CS molecular line observations of Ohashi et al. (1996), is the most likely cause of the additional reddening.

However, the overall picture of TMR-1's protostellar envelope+disk+outflow structure is quite complex and appears undetermined, despite the availability of a number of observations at various wavelengths. What seems certain is that TMR-1AB currently drives a molecular outflow detected previously at different molecular lines, with an outflow position angle of $\sim 170 \pm 10^{\circ}$ (Hogerheijde et al. 1998; Terebey et al. 1990). Red-shifted gas is found north of TMR-1AB, and the NW nebulosity appears to be associated with lower velocity red-shifted gas. Thus, the NW nebulosity and its filament has been interpreted as being part of an outflow cavity. A cavity opening angle of $\sim 30^{\circ}$ was derived from multi-wavelength envelope modeling by Furlan et al. (2008). There is no clear detection of a blue-lobe of the outflow, which would be expected south of TMR-1AB. Most of the blue-shifted gas instead, seems peaked at or close to TMR-1AB, which is roughly at the center of the SE nebulosity. The SE nebulosity, therefore, has been suggested to trace the gravitationally bound circumstellar or circumbinary material close to the protostars. The filament pointing towards TMR-1C, on the other hand, could be part of an outflow cavity rim.

Adding to the complexity, we clearly observe in our deep ISAAC images a striking apparent symmetry between the NW and SE nebulosities, the extending filament arms and the presence of a point-source on either end of the filaments (cf. Fig. 1b, c). The center of symmetry is determined by a large "gap" or lane of 4". 5 in size, i.e. almost $700 \mathrm{AU}$, in-between the two nebulosities (see also, Terebey et al. 1990; Itoh et al. 1999). While in some previous studies the dark lane had been interpreted as an extinction structure, we suggest that the gap is rather devoid of high density material, because neither cold dust emission maps from either single dish or interferometer observations nor other high column density tracers detect the dark lane structure (Motte \& André 2001; Petr-Gotzens et al. 2002; Walsh priv. comm.). The same conclusion was reached by Whitney et al. (1997) based on the relatively blue $H-K$ colour observed towards the gap 

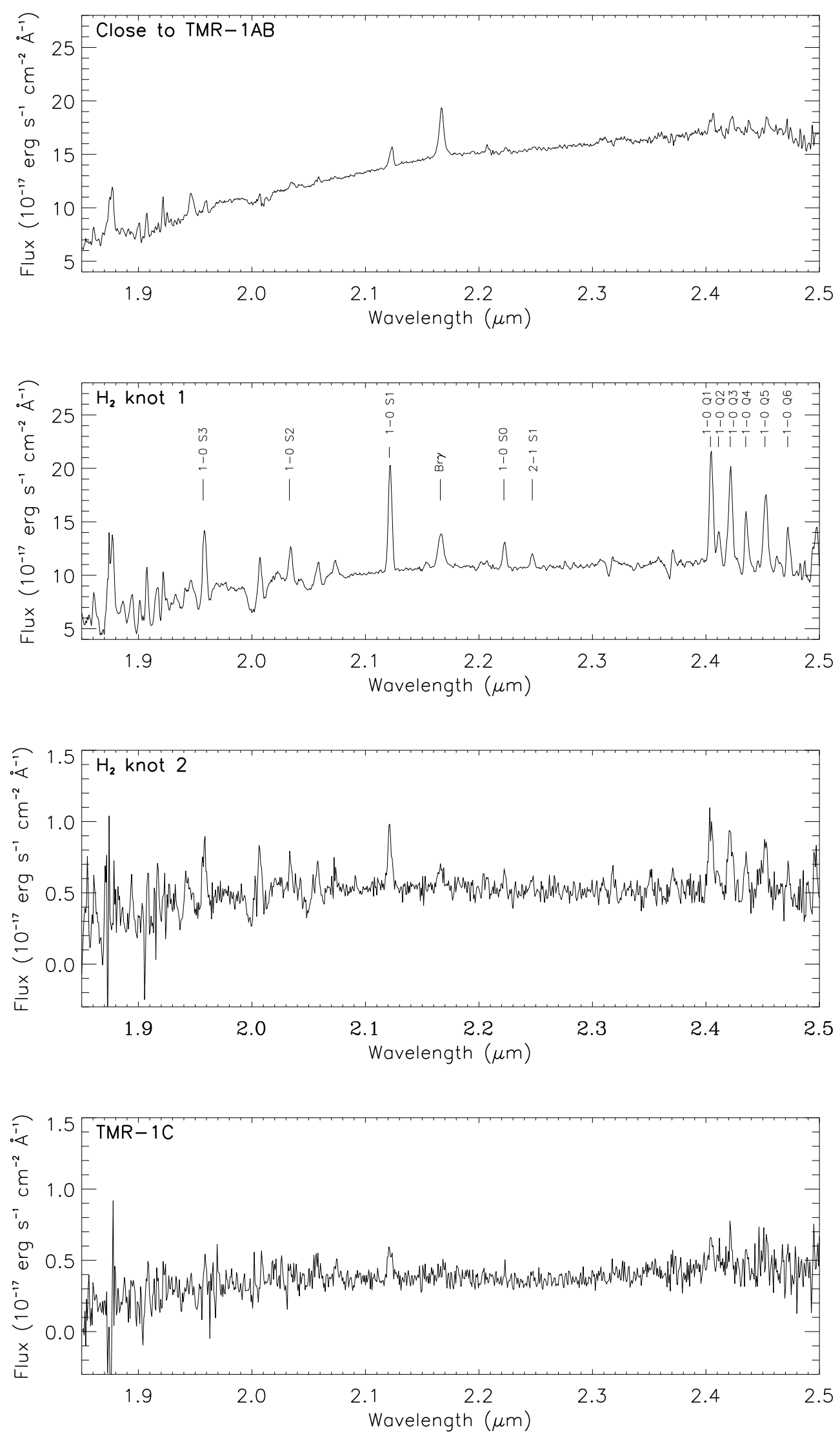

Fig. 4. Extracted TMR-1 spectra. All spectra cover the same wavelength range from $1.85-2.5 \mu \mathrm{m}$. The identification and positions of ro-vibrational $\mathrm{H}_{2}$ lines and the $\mathrm{Br} \gamma$ emission line are indicated in the spectrum of the $\mathrm{H}_{2}$ knot 1. Note that atomic hydrogen emission lines (in particular the $\mathrm{Br} \gamma$-line) are caused or reinforced by division through the standard star, which shows hydrogen absorption (see text for further discussion). 
Table 2. Observed line fluxes in TMR-1 spectra, normalized to the $\mathrm{H}_{2}$ $1-0 \mathrm{~S}(1)$ flux.

\begin{tabular}{|c|c|c|c|}
\hline & $\begin{array}{l}\text { Spectral } \\
\text { line }\end{array}$ & $\begin{array}{c}\text { Measured } \\
\text { wavelength } \\
(\AA)\end{array}$ & $\begin{array}{c}\text { Normalized } \\
\text { integrated line flux }\end{array}$ \\
\hline \multirow{7}{*}{$\begin{array}{l}\text { close to } \\
\text { TMR-1AB }\end{array}$} & $\mathrm{H}_{2} 1-0 \mathrm{~S}(1)$ & 21222 & $1.00 \pm 0.02$ \\
\hline & $\operatorname{Br} \gamma(7-4)$ & 21664 & \\
\hline & $\mathrm{H}_{2} 1-0 \mathrm{~S}(0)$ & 22231 & $0.22 \pm 0.03$ \\
\hline & $\mathrm{H}_{2} 1-0 \mathrm{Q}(1)$ & 24057 & $1.29 \pm 0.29$ \\
\hline & $\mathrm{H}_{2} 1-0 \mathrm{Q}(3)$ & 24226 & $0.86 \pm 0.11$ \\
\hline & $\mathrm{H}_{2} 1-0 \mathrm{Q}(4)$ & 24377 & $0.33 \pm 0.06$ \\
\hline & $\mathrm{H}_{2} 1-0 \mathrm{Q}(5)$ & 24536 & $0.69 \pm 0.09$ \\
\hline \multirow{12}{*}{$\mathrm{H}_{2}$ knot 1} & $\mathrm{H}_{2} 1-0 \mathrm{~S}(3)$ & 19573 & $0.49 \pm 0.01$ \\
\hline & $\mathrm{H}_{2} 1-0 \mathrm{~S}(2)$ & 20332 & $0.34 \pm 0.02$ \\
\hline & $\mathrm{H}_{2} 1-0 \mathrm{~S}(1)$ & 21211 & $1.00 \pm 0.004$ \\
\hline & $\operatorname{Br} \gamma(7-4)$ & 21659 & \\
\hline & $\mathrm{H}_{2} 1-0 \mathrm{~S}(0)$ & 22220 & $0.23 \pm 0.01$ \\
\hline & $\mathrm{H}_{2} 2-1 \mathrm{~S}(1)$ & 22466 & $0.12 \pm 0.002$ \\
\hline & $\mathrm{H}_{2} 1-0 \mathrm{Q}(1)$ & 24044 & $1.06 \pm 0.02$ \\
\hline & $\mathrm{H}_{2} 1-0 \mathrm{Q}(2)$ & 24110 & $0.29 \pm 0.01$ \\
\hline & $\mathrm{H}_{2} 1-0 \mathrm{Q}(3)$ & 24214 & $0.87 \pm 0.01$ \\
\hline & $\mathrm{H}_{2} 1-0 \mathrm{Q}(4)$ & 24353 & $0.46 \pm 0.02$ \\
\hline & $\mathrm{H}_{2} 1-0 \mathrm{Q}(5)$ & 24524 & $0.80 \pm 0.01$ \\
\hline & $\mathrm{H}_{2} 1-0 \mathrm{Q}(6)$ & 24724 & $0.40 \pm 0.01$ \\
\hline \multirow{10}{*}{$\mathrm{H}_{2}$ knot 2} & $\mathrm{H}_{2} 1-0 \mathrm{~S}(3)$ & 19571 & $0.71 \pm 0.03$ \\
\hline & $\mathrm{H}_{2} 1-0 \mathrm{~S}(2)$ & 20328 & $0.26 \pm 0.05$ \\
\hline & $\mathrm{H}_{2} 1-0 \mathrm{~S}(1)$ & 21206 & $1.00 \pm 0.04$ \\
\hline & $\mathrm{H}_{2} 1-0 \mathrm{~S}(0)$ & 22221 & $0.20 \pm 0.01$ \\
\hline & $\mathrm{H}_{2} 2-1 \mathrm{~S}(1)$ & 22465 & $0.18 \pm 0.10$ \\
\hline & $\mathrm{H}_{2} 1-0 \mathrm{Q}(1)$ & 24039 & $1.22 \pm 0.02$ \\
\hline & $\mathrm{H}_{2} 1-0 \mathrm{Q}(2)$ & 24099 & $0.30 \pm 0.07$ \\
\hline & $\mathrm{H}_{2} 1-0 \mathrm{Q}(3)$ & 24208 & $1.14 \pm 0.23$ \\
\hline & $\mathrm{H}_{2} 1-0 \mathrm{Q}(4)$ & 24351 & $0.58 \pm 0.13$ \\
\hline & $\mathrm{H}_{2} 1-0 \mathrm{Q}(5)$ & 24522 & $0.85 \pm 0.11$ \\
\hline
\end{tabular}

Notes. No line fluxes are given for the $\mathrm{Br} \gamma$ line, because the observed fluxes are overestimated (see main text for further discussion).

structure. TMR-1AB appears offset from the gap. This may be a geometrical effect, if TMR-1AB were surrounded, for example, by a large ring of dense gas with an inner hole and the ring being tilted towards the observer. The nebulosities would trace to some extent the optically thick surface of this ring. But without more detailed information about the gas kinematics and observations at multi-wavelengths, no firm conclusion can be made.

In the context of our study of the nature of the point-source TMR-1C, yet the most noteworthy observation revealed by our images is the symmetric location of TMR-1C and TMR-1D, each at the end of a long filament structure. We interpret the morphology as such as if the filament arms had been swept up by a common symmetric outflow. The filaments are not an outflow or jet by themselves, because they are composed of large amounts of continuum emission. Maybe the expansion of a powerful bipolar outflow triggered the collapse of small low-mass cores, leading to the formation of TMR-1C and 1D? Based on our ISAAC images, we measure a position angle of $\sim 145^{\circ}$ for a line tracing roughly the direction of the filaments and connecting TMR-1C with TMR-1D. The position angle of the currently observed molecular outflow, detected at several different molecular lines has been determined to be $\sim 170 \pm 10^{\circ}$ (Hogerheijde et al. 1998; Terebey et al. 1990). One may speculate that the main outflow direction has changed counter-clockwise in the past, because the current outflow's axis seems different from the position angle formed by TMR-1C and 1D. Alternatively, one binary component's outflow has already ceased and we currently witness only the other component's outflow. In this context, we note another intriguing new detection from the deep ISAAC image: clearly visible in Fig. 1a, detected at $1.2^{\prime}$ distance south-southeast of TMR-1, there is a bright arc-like structure of likely swept-up material, whose morphology resembles that of a bow-shock. However, no excessive line emission at $2.12 \mu \mathrm{m}$ is detected, which should be expected if the emission were caused by shock-excitation. The Spitzer/IRAC images at $3.6 \mu \mathrm{m}$ and $4.5 \mu \mathrm{m}$ confirm the structure (Fig. 2), but neither the IRAC images nor our deep ISAAC images show an associated north-northwest counterpart. This is unlikely because of higher extinction, because there is no indication of this, from neither star counts nor dust continuum maps. Although, clumpy extinction on small scales cannot be excluded. If a counterpart feature exists, its emission must be faint. The arc structure may represent a fossil, compressed post-shock region, i.e. a signature from an earlier passage of a shock wave. Its position angle, measured from TMR-1AB to the arc head is $\sim 160^{\circ}$. In Sect. 4, we elaborate on the discussion of possible formation mechanisms for TMR$1 \mathrm{C}$ and TMR-1D, and for the filament structures. Given these new findings, we reconsider in the following sections the background star versus very low-mass object debate for TMR-1C.

\subsection{TMR-1C, a background star...?}

Since TMR-1 is embedded in a region of the Taurus molecular cloud that is heavily obscured by dust, we test the hypothesis that TMR-1C is an unrelated star located behind the molecular cloud that has its light extincted by $A_{\mathrm{v}}>20 \mathrm{mag}$. Low-resolution spectroscopic observations of Terebey et al. (2000) actually showed that the near-infrared spectrum of TMR-1C is not inconsistent with an extincted background dwarf star spectrum.

Our ISAAC spectrum of TMR-1C is shown in Fig. 4. Its $S / N$ is about $8-10$. The interpretation is not straightforward, as the spectrum lacks any spectral features that could be useful for diagnostics. The emission line seen at $2.12 \mu \mathrm{m}$, and those at wavelengths beyond $2.4 \mu \mathrm{m}$ are residuals from molecular emission from the tip of the filament $\left(\mathrm{H}_{2} \mathrm{knot} 2\right)$ and do not originate in TMR-1C itself. The ISAAC spectrum of TMR-1C is very similar to the spectrum obtained at Keck by Terebey et al. (2000), hence does not provide any additional new weight to the background star origin of TMR-1C.

In Fig. 5, we compare our TMR-1C spectrum with an extincted K7V star spectrum from the spectral library of Pickles (1998). The spectral resolution of the library spectra was degraded and smoothed to fit the resolution of the ISAAC spectrum, and noise was added to resemble the $S / N$ of the spectrum of TMR-1C. The amount of extinction to be applied to the dwarf star spectrum was constrained by the $H-K$ colour of TMR-1C. With $H-K=1.65 \pm 0.1$, as implied by our ISAAC photometry and from measurements reported in T98, $A_{\mathrm{v}}$ was constrained to 20-27 mag for normal interstellar dust extinction of the stellar photosphere's of B to early-M type dwarf stars. Applying an extinction equivalent of $A_{\mathrm{V}}=23$ mag to the K7V library star spectrum, leads to a reasonable conformity with the spectrum of TMR-1C as shown in Fig. 5. We, however, note that the comparison was based mainly on the slope of the spectra, because no spectral features are present in TMR-1C's spectrum. As normal stars earlier than M-type do not show a significant difference in their spectral slope over the wavelength range analysed here, the spectral type of TMR-1C, assuming a background dwarf, can only be classified as M 5 or hotter, and the K7V star spectrum that we show in Fig. 5 provides an example of a conceivable fit. 


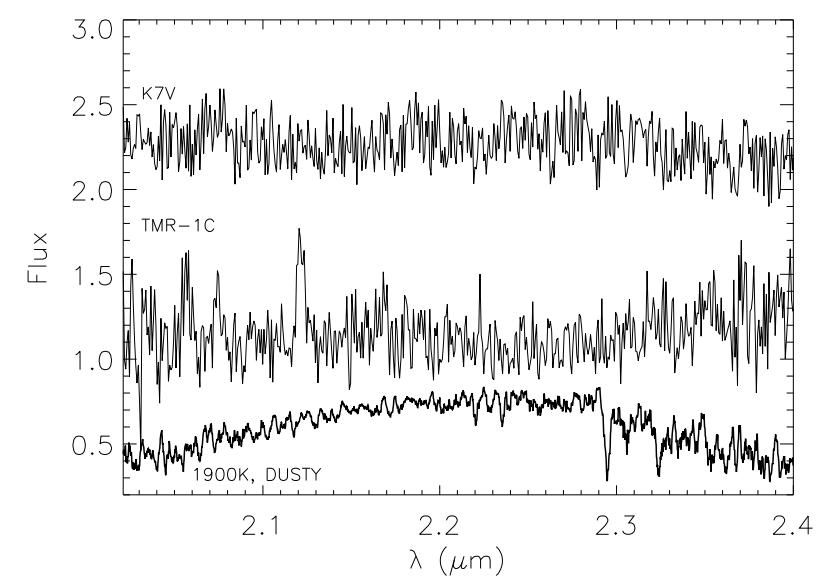

Fig. 5. Upper spectrum: $\mathrm{K} 7 \mathrm{~V}$ star spectrum from the stellar spectral library of Pickles (1998), smoothed and extincted by $A_{\mathrm{v}}=23$ mag, and noise added. Middle spectrum: TMR-1C. Lower spectrum: a $T_{\mathrm{eff}}=$ $1900 \mathrm{~K}$ synthetic spectrum with $\log g=3.5$ from the DUSTY model (Chabrier et al. 2000; Allard et al. 2001). Spectra are in arbitrary flux units, with the K7 V spectrum and the DUSTY model spectrum being offset for comparison.

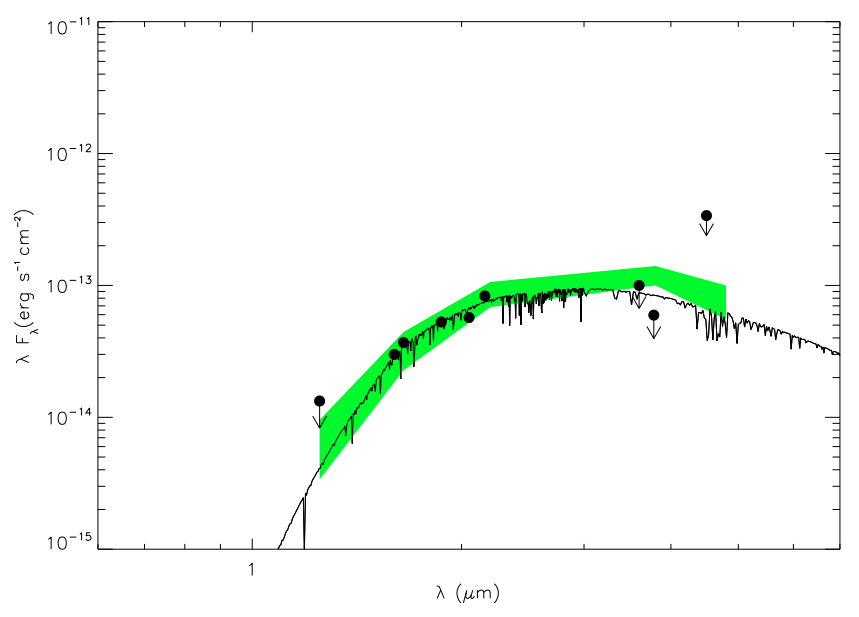

Fig. 6. Observed spectral energy distribution of TMR-1C (filled black circles, based on Table 1 and on values reported in T98; filled black circles with downward-heading arrows indicate upper $3 \sigma$ flux limits). The black solid line represents the spectral energy distribution of a NEXTGen stellar model source (Hauschildt et al. 1999) with $T_{\text {eff }}=$ $4000 \mathrm{~K}, \log g=5.0$ and extincted by $A_{V}=23.0 \mathrm{mag}$. The shaded band indicates the range in flux distribution expected for objects of 3-7 $M_{\text {Jup }}$ at the age of $1 \mathrm{Myr}$ and seen behind $A_{\mathrm{V}}=10-22 \mathrm{mag}$ of extinction, which are consistent overall with the observed SED of TMR-1C for $\lambda<2.5 \mu \mathrm{m}$.

More information about the nature of TMR-1C can be obtained from an analysis of its full spectral energy distribution, as much as is accessible. To do so, we use our photometric measurements and photometry from T 98 to compile the SED of TMR-1C. Then, we compare to model spectra using NextGen stellar atmosphere models (Hauschildt et al. 1999), and find that for wavelengths shorter than $2.5 \mu \mathrm{m}$, the flux distribution of TMR-1C is, for example, represented well by a main-sequence dwarf star with $T_{\text {eff }}=4000 \mathrm{~K}$, seen through $A_{\mathrm{v}}=23 \mathrm{mag}$ of interstellar extinction (Fig. 6). This is consistent with the result shown in Fig. 5. As mentioned already above, combinations of lower values of $A_{\mathrm{v}}$ and $T_{\text {eff }}$, or higher values of $A_{\mathrm{v}}$ with higher $T_{\text {eff }}$ do also lead to a good fit. With an upper limit of $A_{\mathrm{v}}=30 \mathrm{mag}$, a stellar photosphere with $T_{\text {eff }}$ as high as $20000 \mathrm{~K}$ is still in agreement with the observed spectral energy distribution in the near-infrared regime. However, the obviously strong decline in TMR-1C's flux distribution in the mid-infrared, as reflected by its non-detection, is inconsistent with any reddened normal stellar photospheres. We conclude that TMR-1C is not an extincted background star.

Our conclusion is also supported from a purely statistical point of view, because the chances of TMR-1C being a background object projected along the line of sight close to TMR$1 \mathrm{AB}$ are low: we calculate the probability that a background object coincidently falls within $10^{\prime \prime}$ of TMR-1AB, which is the separation between TMR-1C and $1 \mathrm{AB}$. For doing so, we first determine the stellar density of $K$-band sources from our relatively large field ISAAC observations, assuming that all faint objects are background sources. Since we detect 25 stars with a $K$ s-magnitude similar or fainter than that of TMR1C, we derive $\sim 1 \times 10^{-3}$ stars/arcsec ${ }^{2}$, hence a probability of 0.22 of finding a background object as close as $10^{\prime \prime}$ to TMR-1AB. But TMR-1C is not only found somewhere at $10^{\prime \prime}$ distance around TMR-1AB, but at the tip of a narrow filament. We note that the 2D spectrum clearly shows that the molecular hydrogen emission from the filament stops exactly where TMR-1C is located. Consequently, we evaluate the probability of finding a random background star as close as $10^{\prime \prime}$ to TMR-1AB and within $1^{\circ}$ of a certain position angle around TMR-1AB. This probability is only $6 \times 10^{-4}$.

\section{3. ... Or a very low-mass object?}

If TMR-1C is not a chance projected background star, what kind of object is it? Since the spectrum is obviously pure continuum it is consistent with neither a bullet nor dense knot of shocked molecular gas. Moreover, for all imaging observations reported so far, TMR-1C appears as an unresolved point-source; the HST image even shows the first Airy diffraction ring.

The study of T98 proposed that if TMR-1C were physically associated with TMR-1AB it should be a young sub-stellar object, since it is several magnitudes less luminous than TMR$1 \mathrm{AB}$, which is a proto-stellar system of $\sim 0.5 M_{\odot}$ (Kenyon et al. 1993, T98). TMR-1C's bolometric luminosity could be as low as $10^{-3}-10^{-4} L_{\odot}$ as estimated by T 98 and Terebey et al. (2000). In Fig. 6, we plot, as a shaded band, the range of SEDs for planetary mass objects with 3-7 $M_{\text {Jup }}$ and of the age 1 Myr (based on DUSTY and COND sub-stellar evolutionary models of Chabrier et al. 2000; Baraffe et al. 2003) that are consistent with the nearinfrared SED of TMR-1C. Depending on the mass of the object, and hence on the effective temperature, different values of extinction, ranging from $A_{\mathrm{v}}=10-22 \mathrm{mag}$ had to be added to achieve a fit with the near-infrared part of the observed SED of TMR-1C. While the general slope of TMR-1C's near-infrared spectral energy distribution is consistent with extincted photospheres of 3-7 $M_{\text {Jup }}$ mass objects, the expected value of effective temperatures for these low-mass objects $(<2100 \mathrm{~K})$, however, is in strong contradiction with our observed $K$-band spectrum that indicates higher $T_{\text {eff }}(\gtrsim 3000 \mathrm{~K})$. $K$-band spectra of cool objects with effective temperatures $<2500 \mathrm{~K}$ display prominent signatures in the $K$-band, such as deep methane and/or water vapor absorption bands, the $v=2-0$ vibration-rotation bands of $\mathrm{CO}$ at $\lambda>2.29 \mu \mathrm{m}$, and the $\mathrm{NaI}$ atomic absorption line at $2.21 \mu \mathrm{m}$ (Burrows et al. 2001, and references therein). In general, the depth of the $\mathrm{H}_{2} \mathrm{O}$ and $\mathrm{CH}_{4}$ absorption increases from M-class to L- and T-class objects, though at some phase during 
$\mathrm{L}$ spectral class $\mathrm{H}_{2} \mathrm{O}$ absorption is weakened by dust formation and re-radiation. But in any case, the molecular absorption is so pronounced, especially for the coldest very low-mass T-dwarfs, that it is easily detectable even in low signal-to-noise spectra (e.g. Cuby et al. 1999). It appears that these strong absorption bands are also present in young $(\leq 1 \mathrm{Myr})$, low gravity, low-mass objects. For instance, distinct water vapor absorption bands have been observed in near-infrared spectra of brown dwarfs and planetary mass objects in the very young $\rho$ Oph and Orion Trapezium cluster (Wilking, et al. 1999; Lucas et al. 2001; Lucas et al. 2006). None of the features described above are present in our spectrum of TMR-1C. In Fig. 5, we show for comparison the synthetic spectrum of a young $5 M_{\text {Jup }}$ object with $T_{\text {eff }}=1900 \mathrm{~K}$ and $\log g=3.5$. This model spectrum, generated by the Lyon group based on the Ames-DUSTY stellar evolutionary model (Chabrier et al. 2000; Allard et al. 2001), is clearly different from the spectrum of TMR-1C. Furthermore, as implied by Fig. 6, any young planetary mass object should be brighter than our measured upper detection limits at mid-infrared wavelengths.

An alternative solution may be that TMR-1C is physically associated with TMR-1AB, but instead of being an extremely low-mass object, it may be a slightly higher mass object whose light is dimmed significantly by a surrounding circumstellar disk seen edge-on. In this case, the emergent flux in the near-IR is dominated by scattered light from the disk surface. TMR-1C, if occulted by a disk, would appear subluminous for its spectral type and may exhibit an observed near-IR spectral energy distribution that differs from the object's intrinsic colours. This hypothesis for the nature of TMR-1C was previously proposed by Hartmann et al. (1999). Direct and indirect evidence of disks around young substellar objects have been found by several authors (e.g. Natta et al. 2002; Luhman et al. 2007). These studies show that disks around young brown dwarfs have properties that are, in general, similar to those derived for circumstellar disks of low-mass stars. In addition, low-resolution near-IR spectra of objects with edge-on disks often appear featureless (e.g. Brandner et al. 2000; Scholz et al. 2008), as in the case of the TMR-1C spectrum. To explore this possibility, we used the SED model fitting tool ${ }^{3}$ of Robitaille et al. (2007), which was developed to analyze the SEDs of young stellar objects. A particular advantage of this tool is that it allows the inclusion of upper flux limits. To limit the solutions to models that are physically relevant to TMR-1C, we postulate that the source's distance must be within $117-157 \mathrm{pc}$, the range in distance considered for the Taurus molecular cloud (Torres et al. 2007), and that its age must be $\lesssim 1 \mathrm{Myr}$. The model that is then in closest agreement with the observed SED of TMR-1C is shown in Fig. 7. The model SED shows a steep decline beyond $2.2 \mu \mathrm{m}$, and a strong excess at $\lambda>10 \mu \mathrm{m}$. The underlying model is that of a central source of $\sim 0.13 M_{\odot}$ at a distance of $138 \mathrm{pc}$, with the parameters of age of the source $\sim 4 \times 10^{5} \mathrm{yr}, A_{\mathrm{v}}=18 \mathrm{mag}, T_{\text {eff }}=3000 \mathrm{~K}$, inclination angle of the circumstellar disk, $i=87^{\circ}$, inner disk radius, $r_{i}=1.8 \mathrm{AU}$, and disk outer radius, $r_{\mathrm{o}}=94 \mathrm{AU}$. The predicted size of the disk seems large, given that TMR-1C appears unresolved in all the HST and ISAAC images. An edge-on disk as large as $r_{\mathrm{O}}=94 \mathrm{AU}$ and its associated bipolar scattered light structures should have appeared resolved in the high spatial resolution HST images ( $\left.F W H M \sim 00^{\prime} 15-0.2\right)$ and possibly also in the highest quality ISAAC images. The fact that TMR$1 \mathrm{C}$ appears as a point-source implies that the actual disk must be smaller than $\sim 20 \mathrm{AU}$. A decrease in the outer disk size will

3 http://caravan.astro.wisc. edu/protostars/sedfitter. php

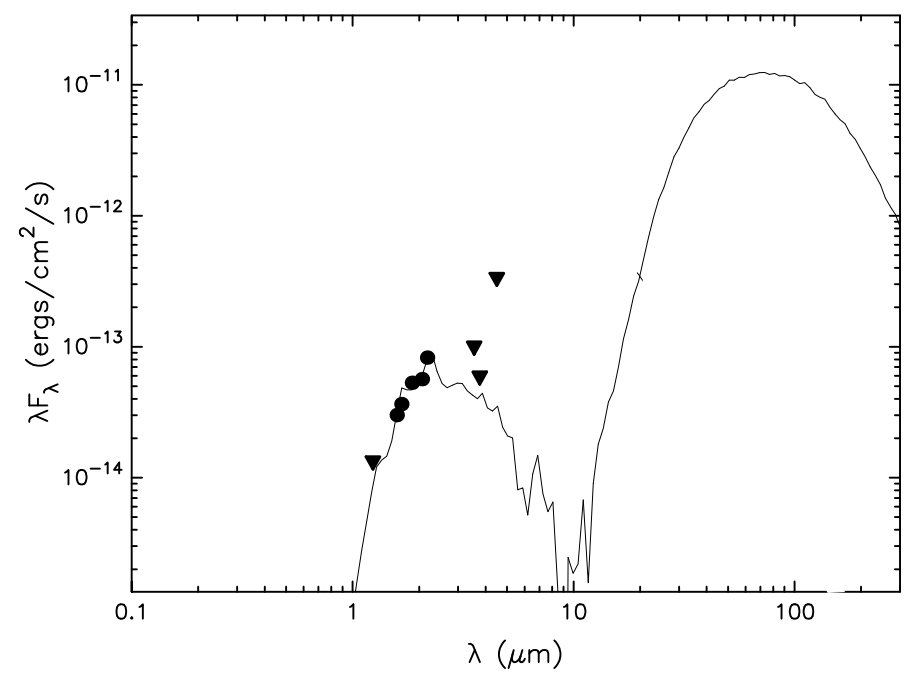

Fig. 7. Spectral energy distribution of TMR-1C (indicated by filled dots, triangles represent upper limits) compared to a model SED for a central source of $0.13 M_{\odot}$ with a circumstellar disk inclined to our line of sight by $i=87 \mathrm{deg}$, an outer disk radius of $94 \mathrm{AU}$, an age of $\approx 4 \times 10^{5} \mathrm{yr}$, and located at a distance of $138 \mathrm{pc}$ seen through an interstellar extinction of $A_{V}=18.0 \mathrm{mag}$.

mostly affect TMR-1C's spectral energy distribution longward of $10 \mu \mathrm{m}$, i.e. in a regime that is unconstrained because of a lack of observations. In a picture where TMR-1C was ejected in a dynamical interaction within the small proto-stellar cluster of TMR-1, it is expected that any primordial disk of TMR-1C was truncated (cf. Sect. 4). We note that if one considers models for sources at slightly larger distances (up to $\sim 170 \mathrm{pc}$ ) or slightly older objects (up to $\sim 1.2 \times 10^{6} \mathrm{yr}$ ), one finds that also objects with $\sim 0.2-0.4 M_{\odot}$ and disk sizes as small as 19 AU provide reasonable fits to the SED of TMR-1C. However, since the SED is unconstrained beyond $5 \mu \mathrm{m}$, it is currently not possible to distinguish more clearly between the model solutions. SED models for objects with central source masses $<0.1 M_{\odot}$ are not available. However, we emphasize that all models providing a good fit indicate a circumstellar disk with high inclination angle of $i>80^{\circ}$.

Observational evidence of an edge-on disk surrounding TMR-1C would be an infrared excess emission at $\lambda>10 \mu \mathrm{m}$. Unfortunately, the spatial resolution of available observations at mid to far-infrared wavelengths are too low to differentiate potential emission from TMR-1C from the emission of TMR-1AB. Other evidence, as discussed above, could be provided by nearinfrared images with high sensitivity and a spatial resolution higher than 0 .' 1 that should reveal any resolved bipolar scatteredlight structure.

\subsection{On the nature of the filament}

It has been suggested that the arc-shaped filamentary structure that seems to "connect" TMR-1C with TMR-1AB is a material tail formed during an encounter of proto-stellar disks surrounding TMR-1A and B (Lin et al. 1998, T98). The same encounter may have caused the formation of TMR-1C by means of fragmentation of a part of the filament into a very low-mass object, or ejection during the dynamical interaction. In these cases, there would be a clear physical relationship between the filament and TMR-1C. 


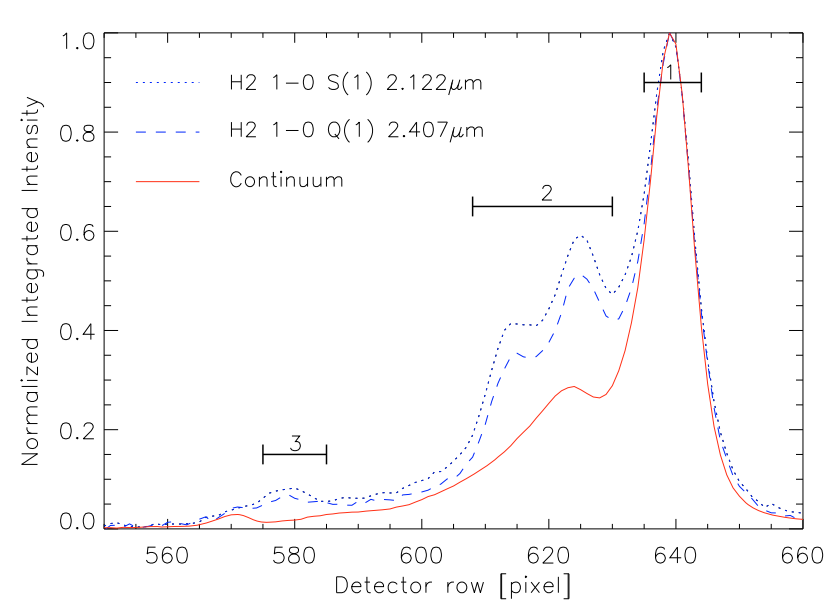

Fig. 8. Spatial distribution of the intensities in the $\mathrm{H}_{2}$ emission lines (dotted and dashed blue lines) along with the continuum (red solid line). The intensities have been normalized to their peak emission in the ISAAC TMR-1 spectrum. The apertures of extracted spectra are also indicated: (1) close to TMR-1AB, (2) $\mathrm{H}_{2}$ knot 1, (3) $\mathrm{H}_{2}$ knot 2.

The higher spectral resolution of our ISAAC data compared to the previous spectrum of the filament (Terebey et al. 2000) allows us to extract new physical information about the nature of the filament. Previous studies mainly reported on the scattered light emission arising from the filament. However, in spite of a clear component of continuum emission, we propose that there is another distinct process present, that is shocks. In Fig. 8, we plot the spatial distribution of the intensities in the $\mathrm{H}_{2} 1-0 \mathrm{~S}(1)$ and $\mathrm{H}_{2} 1-0 \mathrm{Q}(1)$ emission lines, and in the continuum, along the filament. There are obviously 2 locations where the emission in molecular hydrogen is significantly in excess. Spectra at these 2 locations along the filament have been extracted $\left(\mathrm{H}_{2}\right.$ knot 1 and $\mathrm{H}_{2}$ knot 2 in Fig. 4, note the $\mathrm{H}_{2}$ knot 1 location, i.e. aperture 2 , actually includes 2 peaks of $\mathrm{H}_{2}$ emission which show indistinguishable spectra, which were therefore combined to improve the signal-to-noise).

Both spectra exhibit a wealth of emission lines, which are predominantly ro-vibrational lines of molecular hydrogen.

Molecular hydrogen emission originates mainly in either of the two physical processes: shock excitation or ultraviolet fluorescence. We use the intensity ratio of the transitions $\mathrm{H}_{2}$ $v=1-0 \mathrm{~S}(1)$ at $2.12 \mu \mathrm{m}$ and $\mathrm{H}_{2} v=2-1 \mathrm{~S}(1)$ at $2.24 \mu \mathrm{m}$ to distinguish between these two cases. Thermal excitation via shocks should be the mechanism responsible if this ratio is $\gtrsim 5$, and gas densities are not too high, i.e. $\lesssim 10^{5} \mathrm{~cm}^{-3}$ (Shull \& Beckwith 1982). Since we measure a $2.12 \mu \mathrm{m} / 2.24 \mu \mathrm{m}$ ratio of $\sim 8.25$ and $\sim 5.6$, for the $\mathrm{H}_{2}$ knot 1 spectrum and the $\mathrm{H}_{2}$ knot 2 spectrum, respectively, and we do not observe any lines from transitions of high vibrational levels, we conclude that the $\mathrm{H}_{2}$ emission in the filament is due to shock excitation. Support for the shock interpretation is also gained from $\mathrm{H}_{2}$ ortho-para ratios discussed below.

The situation is slightly different for the spectrum extracted close to TMR-1AB, which does not show any detectable emission at $2.24 \mu \mathrm{m}$, suggesting the absence of molecular gas at higher temperatures $(\gtrsim 2000 \mathrm{~K})$. The $\mathrm{Br} \gamma$ recombination line of atomic hydrogen at $2.166 \mu \mathrm{m}$, on the other hand, is one of the most prominent lines in this spectrum. This indicates that a process must be present that is capable of ionizing hydrogen. Winds from the young stellar object is one of the likely responsible mechanisms. But disk accretion onto the central source may also be the cause of the $\operatorname{Br} \gamma$ emission. Prato et al. (2009) present a $K$-band spectrum of TMR-1AB containing similar features as our ISAAC spectrum obtained close to TMR$1 \mathrm{AB}$ (we recall that the ISAAC slit positioning is offset by almost $1^{\prime \prime}$ from the TMR-1AB peak emission). From the Br $\gamma$ emission line luminosity, these authors determine a mass accretion rate of $1.9 \times 10^{-7} M_{\odot} / \mathrm{yr}$ for TMR-1, which is quite consistent with $\mathrm{Br} \gamma$ emission being generated by accretion.

What causes the shocks in the filament? It has long been known, that TMR-1 is associated with a large molecular outflow (Terebey et al. 1990; Hogerheijde et al. 1998) observed e.g. at ${ }^{12} \mathrm{CO}(3-2)$. The direction of this outflow is in very good agreement with the direction of a jet indicated in FeII narrow-band near-infrared images (Petr-Gotzens et al. 2002). The position angle of the filament differs by $\sim 20^{\circ}$ from the position angle of the outflow and jet. A plausible scenario is that the filament is part of the edge of a cavity cleared by a lower velocity outflow, as for example observed in HH 211 (Gueth \& Guilloteau 1999). The molecular hydrogen emission knots along the filament would then be produced, because this lower velocity flow would collide with the cavity rim, thereby creating a C-type shock (see Sect. 3.4.1). The filament may otherwise be an intrinsic, preexisting dense material shocked by the outflow as it happens to be in its way. It is unlikely that the filament is a jet in itself. While bent jets have been observed (Davis et al. 1994; Bally \& Reipurth 2001), the significant amounts of continuum emission coming from the filament, together with the high degree of polarization (Whitney et al. 1997), and detection of high column densities associated with the filament (Hogerheijde et al. 1998; Motte \& André 2001), exclude a jet.

\subsubsection{A shock-model for the emission from the filament}

While we could establish that the $\mathrm{H}_{2}$ emission along the filament is caused by shocks, the evaluation of the precise details of which shock excitation mechanism is at work, and what the underlying shock physics are is more complex. A powerful method for analyzing different possible mechanisms is to compare the measured column density ratios (CDRs) to various shock excitation models in a schematic CDR diagram. This approach was taken in this study, and we converted our flux measurements of the ro-vibrational molecular hydrogen lines (Table 2) into Column Density Ratios following the calculations given in Eislöffel et al. (2000) and Smith (2000). Thus, we determine the column of $\mathrm{H}_{2}$ in the upper energy level necessary to generate each line flux. In the CDR diagrams, we compare column density ratios to those of a slab of gas of constant temperature $(2000 \mathrm{~K})$. By removing the exponential Boltzmann factor in this way, we are able to analyse the results in detail.

The CDR diagrams for each position along the filament are shown in Figs. 9-11. The shock model plotted on all diagrams is a C-type bow shock. The sparesness of the data, in particular with respect to the range in temperatures, and their scatter prevent detailed fits. We note that a constant temperature model (corresponding to a linear fit) would be consistent with all the data. A horizontal line on the CDR diagrams would correspond to $2000 \mathrm{~K}$. A possibility is a single C-type shock in which the emission originates in an extended zone with a quite narrow range of gas temperatures (Smith \& Brand 1990).

However, shock waves of all types do not maintain gas at a fixed temperature and we expect a range of temperatures to be present. As an example, we demonstrate in the diagrams that at all locations a gently curved shock with some range of shock 

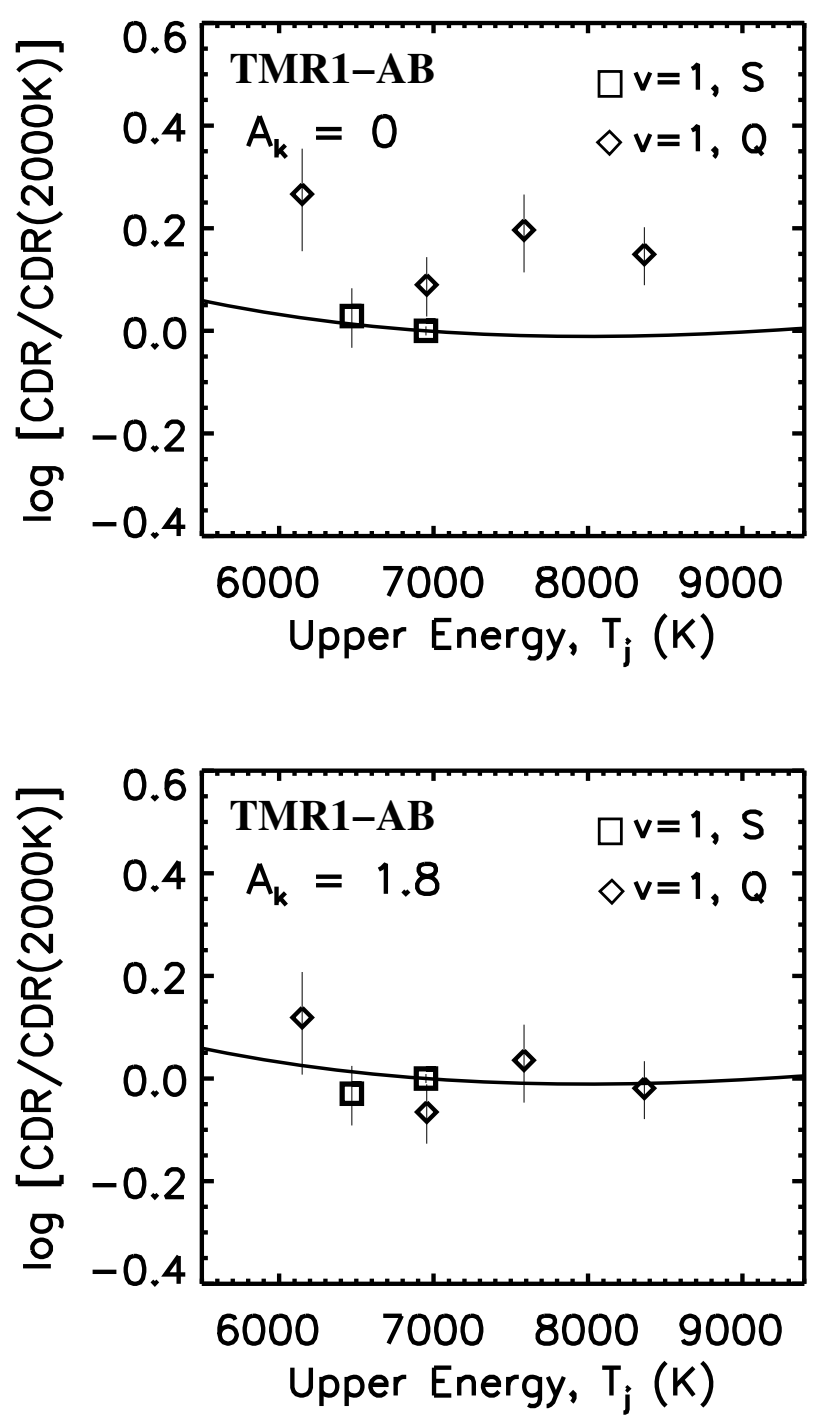

Fig. 9. $\log (\mathrm{CDR})$ diagram derived for the position close to TMR-1AB. The solid line represents a C-type bow shock model.

velocities is also consistent. This issue can be resolved by observing pure rotational lines from low levels in the mid-infrared since very high CDR values are expected from bow shocks (see lower panels of Figs. 10, 11).

All CDR diagrams display no evidence of ortho-para ratios deviating from that usually associated with shock waves. Fluorescent emission from unshocked cloud gas in star formation regions yields enhanced columns of gas in the para upper energy levels (e.g. Li et al. 2008). Here, however, we find that, within the errors, there is no deviation from a thermalised orthopara ratio of 3 (Smith et al. 1997), corresponding to linear fits in the diagrams (note the large errors associated with the noisy parts of the high Q-branch and high S-branch transitions).

\subsubsection{Extinction along the filament}

Estimates of the interstellar extinction can be directly derived from line flux ratios of the Q-branch at 2.4-2.5 $\mu \mathrm{m}$, independent of the specific shock model.

In Fig. 9, we show the data for the filament position close to TMR-1AB with 2 different extinctions (upper and lower panel
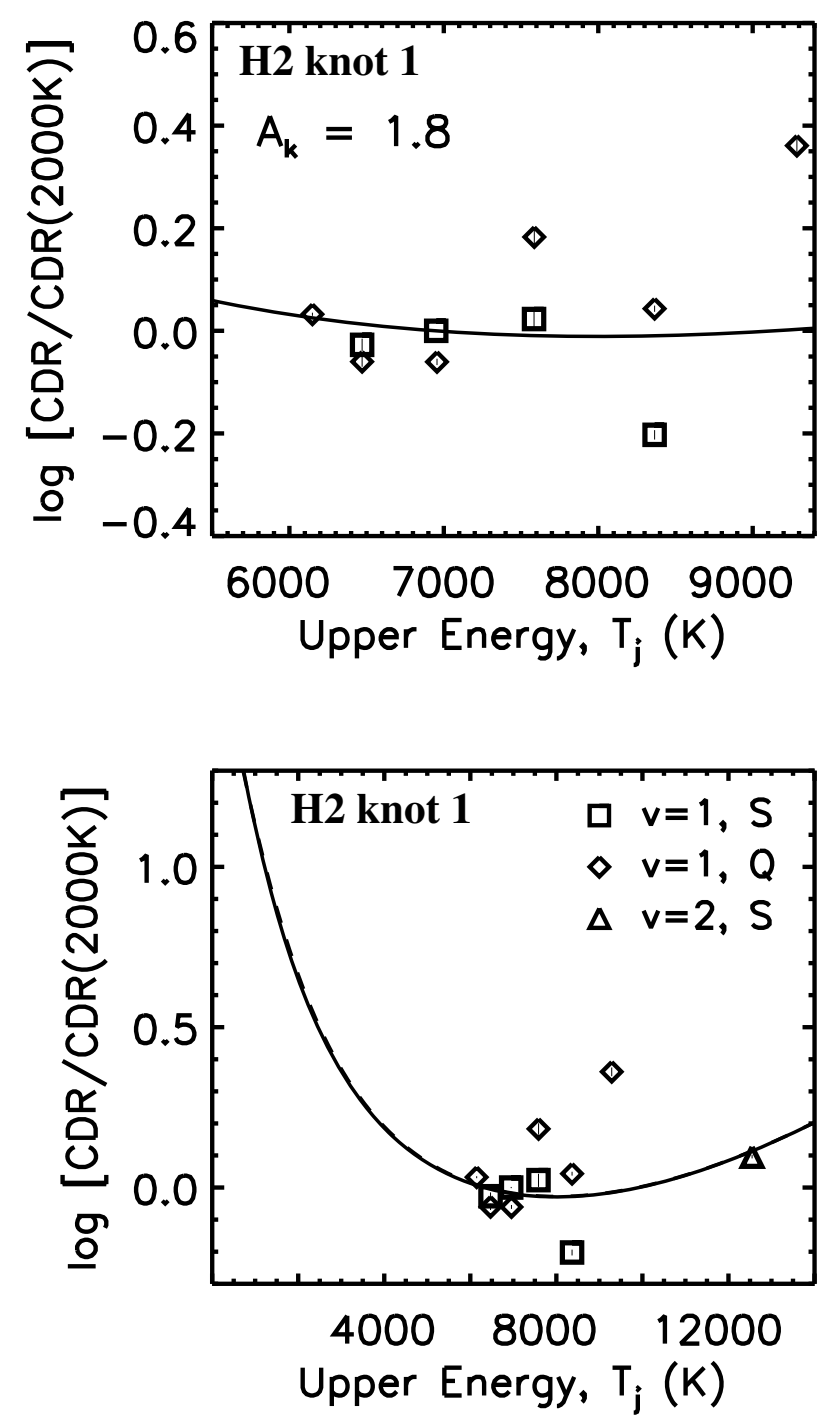

Fig. 10. $\log (\mathrm{CDR})$ diagram derived for the $\mathrm{H}_{2}$ knot 1 position. The solid line represents a C-type bow shock model. The upper panel and lower panel show the same datapoints, with the exception that the lower panel has a wider range of energies, and includes the measurement at $\mathrm{H}_{2} 2-1 \mathrm{~S}(1)$.

of the figure). With $A_{K}=0 \mathrm{mag}$, all the Q-branch datapoints lie above the $\mathrm{S}$-branch datapoints, while a much closer fit to all datapoints with the model is achieved for $A_{K}=1.8 \mathrm{mag}$ (lower panel). The uncertainty in the derived extinction is $\pm 0.3 \mathrm{mag}$. This value can be compared with the extinction determinations toward TMR-1AB reported in Prato et al. (2009) based on lowresolution $K$ - and $L$-band spectra. They find $A_{\mathrm{V}}=(24.2 \pm$ 1.1) mag using an optical depth measurement of the ice absorption feature at $3.1 \mu \mathrm{m}$, and $A_{\mathrm{V}}=(20.8 \pm 1.8)$ mag based on the source's near-infrared colours. Both extinction values are higher than our extinction derived from the molecular hydrogen line ratios. This is not surprising as the different methods trace different lines of sight through the material causing the extinction. In particular, the molecular hydrogen emission lines are supposedly produced in shocked regions away from the protostellar photosphere. Our ISAAC spectrum is also slightly off center $\left(\sim 1^{\prime \prime}\right)$ from the TMR-1AB protostar system, while Prato et al. (2009) centered their spectrum on TMR-1AB. 

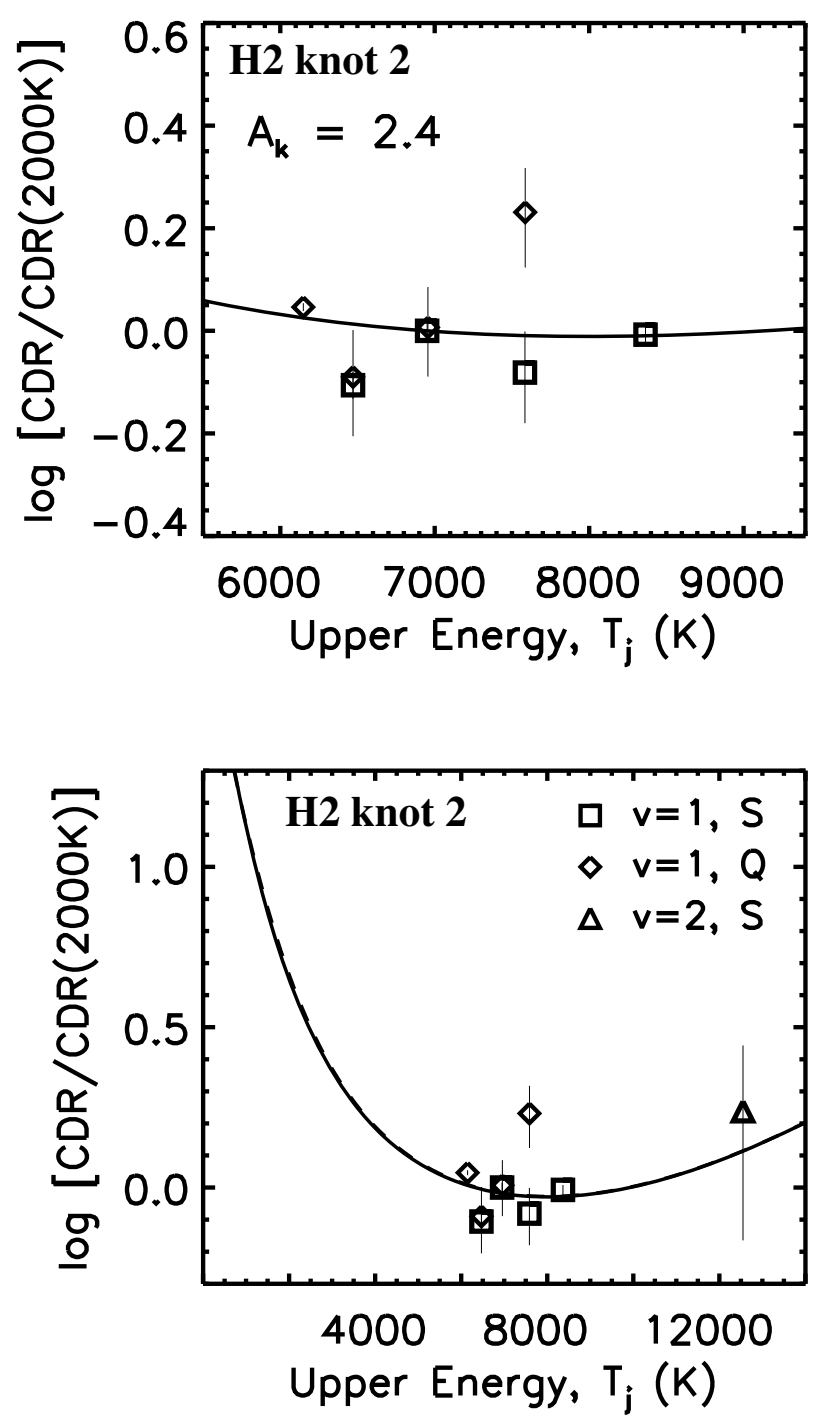

Fig. 11. $\log (\mathrm{CDR})$ diagram derived for the $\mathrm{H}_{2}$ knot 2 position, close to TMR-1C. The solid line represents a C-type bow shock model. The upper panel and lower panel show the same datapoints, with the exception that the lower panel has a wider range of energies, and includes the measurement at $\mathrm{H}_{2} 2-1 \mathrm{~S}(1)$.

For the filament positions $\mathrm{H}_{2}$ knot 1 and $\mathrm{H}_{2}$ knot 2 (Figs. 10 and 11) the modeling is aided by the $\mathrm{H}_{2} 2-1 \mathrm{~S}(1)$ line, enabling a fit over a wider range in temperature. The calculated extinction at the position of the $\mathrm{H}_{2}$ knot 1 is $A_{K}=1.8 \mathrm{mag}$, hence not different from the extinction we determined closer to TMR-1AB. At the position of the $\mathrm{H}_{2}$ knot 2, only $\sim 1^{\prime \prime}$.3 away from TMR-1C, we measure an $A_{K}$ of $2.4 \mathrm{mag}\left[A_{\mathrm{V}}=(21.4 \pm 2.7) \mathrm{mag}\right]$, which is in good agreement with the values of extinction estimated towards TMR-1C (cf. Sects. 3.2 and 3.3). Moreover, the extinction determined at the $\mathrm{H}_{2}$ knot 2 is significantly higher than at the $\mathrm{H}_{2}$ knot 1 and close to TMR-1AB, indicating that the extinction increases from close to the main source along the filament. This finding seems to be in contradiction with the conclusion reached by T98 who observe a higher flux ratio with decreasing wavelength for TMR-1C relative to the protostars TMR-1AB and conclude that there is less extinction towards TMR-1C than towards TMR-1AB. However, TMR-1C could be located in front of the filament, while the filament is indeed receding into the denser parts of the molecular cloud.

If the continuum in the spectrum taken close to TMR-1AB were dominated by the continuum from the protostars, then the spectra continuum slopes (cf. Fig. 4), in principle, would imply a significantly lower interstellar extinction towards TMR$1 \mathrm{C}$ than towards TMR-1AB. Our SED analysis in Sect. $3.3 \mathrm{im}-$ plies $A_{\mathrm{V}}=18.0$ mag for TMR-1C, and Prato et al. (2009) derive $A_{\mathrm{V}}=(24.2 \pm 1.1) \mathrm{mag}$ for TMR-1AB. In addition, strong excess emission from accretion and the (unknown) circumstellar disk geometry contribute to the continuum spectral shape of TMR-1AB. Disentangling the different effects is beyond the scope of this paper.

\section{Formation scenarios for TMR-1C (and TMR-1D)}

We now discuss different models that may qualitatively explain the formation of TMR-1C as a low-mass object physically associated with the proto-binary TMR-1AB. In this picture of the TMR-1 system, TMR-1C and TMR-1D are both very low mass objects (either low-mass stars or brown dwarfs) and their symmetric locations on opposite sides of the main source TMR-1AB shall be taken into account when considering possible formation scenarios.

A scenario worth debating is that of outflow triggered star formation (Sandell \& Knee 2001). This mechanism naturally yields collapsed objects within the high-pressure region associated with a jet or outflow impact. We assume that a string of well-spaced pre-stellar cores existed in this region before one of them collapsed to form the binary system. Provided that the developing proto-stellar jets are directed along the axis connecting the cores, the subsequent impact could trigger a collapse in adjacent cores simultaneously on either side of the expanding outflow. Once the low-mass core is forced into collapse the formation of a low-mass object proceeds "as normal", i.e. its mass continues to grow via disk accretion. This model has the advantage of generating symmetric collapsed objects on opposite sides at the locations of adjacent cores in the string. Moreover, the ongoing outflow may even drive away extended disk and envelope material, generating swept-up dust ridges and shells. The filament would represent denser material along the outflow direction, possibly consisting of swept-up matter, that scatters light having escaped from the main source TMR-1AB through a low density cavity excavated by the jet/outflow. At the densest knots along the filament, the outflow impact gives rise to shock excited emission. Low-mass pre-stellar cores may also be produced in the shock-compressed layers of swept-up dusty filaments (Padoan \& Nordlund 2004). Sandell \& Knee (2001), for example, detect several compact mm-sources in a curving arc of dust near the proto-stellar object SSV13 in the NGC 1333 starforming region.

Intriguingly, the protostellar outflow $\mathrm{HH} 211$ also possesses two symmetric star-like objects at the tips of the presently visible outflow (see Fig. 1 of Eislöffel et al. 2000). As with the TMR-1 outflow, the near-infrared $\mathrm{H}_{2}$ outflow of $\mathrm{HH} 211$ raises curiosity because it also contains a filament of material with strong continuum emission (O'Connell et al. 2005).

Another possibility to discuss is the formation of TMR-1C (and TMR-1D) from disk fragmentation processes. Numerical simulations published by Stamatellos \& Whitworth (2009) show that a number of very low-mass objects with masses in the range $5-200 M_{\mathrm{J}}$ can be produced by gravitational disk fragmentation occurring in an extended circumstellar disk. Most of the produced objects are brown dwarfs $\left(>13 M_{\mathrm{J}}\right)$ and low-mass 
hydrogen-burning stars. Only $\sim 10^{4}$ yrs after the formation of these objects from disk fragmentation, dynamical $N$-body interactions lead to an ejection of most them, i.e. making them unbound and liberate them into the field, but a few objects typically remain bound. Quite interestingly, the most common (i.e. the most likely) configuration resulting from the simulations after a few hundred thousand years is that of a solar-like primary with a nearby low-mass stellar companion ("TMR-1AB"), and two wide $(r>100 \mathrm{AU})$ companions, consisting of a low-mass star ("TMR-1C") and a brown dwarf ("TMR-1D"); this configuration very closely resembles what we conclude we observe for the TMR-1 system. In this model, TMR-1C and TMR-1D remain weakly bound to TMR-1AB. They are also expected to retain their circumstellar disks, although at relatively small sizes, as the disks may had been stripped off even further during dynamical interactions. Dynamical interactions are also held responsible for inhomogeneous orientations of the young objects' circumstellar disks. Non-coplanarity is frequently observed in the modelling (Stamatellos \& Whitworth 2009). It seems plausible that a close passage of, e.g. TMR-1C to TMR-1AB, early in the evolutionary history of the system, caused the circumstellar disk and hence the jet of TMR-1A (or B) to precess, which is what our observations of a bow structure detected at $>1^{\prime}$ distance from TMR-1 indicate.

A shortcoming of the "disk fragmentation with follow up ejection" scenario for the origin of TMR-1C and D is that it does not explain the existence of symmetric filaments and a physical relation of those to TMR-1C and TMR-1D. Although filamentary patterns of the fragmenting primordial disk are observed in the simulations, they are usually short-lived and much smaller in size than what is observed for TMR-1. Therefore, we consider for a moment that both the primary and secondary components of TMR-1AB originally condensed out of the collapsing pre-stellar core, and that both were accompanied by their own extended protostellar disk. In a subsequent encounter of the systems, one assumes that tidal structures consisting of disk material developed ("the filaments"), and that one proto-stellar component was captured by the more massive component to form the proto-binary TMR-1AB. The tidal filaments naturally form symmetric structures. As frequently observed in different numerical simulations, very low-mass objects are then formed out of the densest parts of the filamentary tidal arms (Shen \& Wadsley 2006; Lin et al. 1998), often far from the main source. In this way, there would be a clear causal dependence for the filaments and the candidate low-mass objects TMR-1C and 1D. Shen \& Wadsley (2006) further show that the formed "proto browndwarfs" possess circumstellar disks of sizes up to $\sim 20 \mathrm{AU}$. The objects have typical masses in the range $2-73 M_{\mathrm{J}}$, but the simulations are stopped before mass growth through disk accretion could be completed, so that the objects' final masses may be slightly higher.

\section{Summary and conclusions}

We have used VLT/ISAAC infrared imaging and spectroscopy data, complemented with near-infrared HST and Spitzer/IRAC photometry, to study the nature of low-luminosity objects detected in the close surroundings of the young stellar object TMR1 (actually a binary source: TMR-1AB). We have also discussed the particular morphology of the dust distribution around TMR$1 \mathrm{AB}$ as it appears in the near-infrared images, and how the specific dust structures may be related to a low-mass star formation process. Our main objective has been to place additional constraints on the potential sub-stellar nature of the faint source
TMR-1C, which is a wide companion to the young proto-stellar binary TMR-1AB. The remarkable location of TMR-1C at the tip of a narrow filament emanating from the nebulosity surrounding TMR-1AB indicated a physical association and a substellar nature for TMR-1C in previous studies (T98). Our most important results derived from the analysis in this paper are summarized as follows:

- The near-infrared $K$-band spectrum of TMR-1C is featureless, and fully consistent with previous lower resolution spectra. The spectrum indicates that TMR-1C's effective temperature is $\gtrsim 3000 \mathrm{~K}$ and rejects the possibility of a very cold low-mass object.

- The near to mid-infrared spectral energy distribution of TMR-1C clearly shows a decline in flux for $\lambda>2.5 \mu \mathrm{m}$. It can be fitted by neither an extincted background dwarf star, nor a low-mass brown-dwarf or planetary mass object photosphere, for which the object should have been detected at mid-infrared wavelengths at the sensitivity level of our data.

- The shape of the SED of TMR-1C is consistent with a model SED of a very low-mass stellar source of $0.13 M_{\odot}$ that has a circumstellar disk seen almost edge-on $\left(i=87^{\circ}\right)$ and located at the distance of the Taurus molecular cloud. The presence of the edge-on disk that significantly dims the central source, and of additional interstellar extinction of $A_{\mathrm{V}} \sim 18 \mathrm{mag}$ are then the causes of the very low apparent brightness of TMR$1 \mathrm{C}$. Hence, the observations at near-infrared mainly detect TMR-1C in scattered light.

- In our very sensitive $H$ - and $K$ s-band observations with very good spatial resolution, we were able to detect a faint pointsource that we call TMR-1D ( $K \mathrm{~s} \sim 18.7 \mathrm{mag})$. TMR-1D is located roughly $20^{\prime \prime}$ northwest of TMR-1AB and, in analogy with TMR-1C, is seen at the end of a diffuse filamentlike dust structure originating in the northwestern nebulosity associated with TMR-1. There is an apparent symmetry of TMR-1C and "its filament" with TMR-1D and "its filament". TMR-1D is a very low luminosity object, if it is physically associated with TMR-1.

- The candidate low-mass objects TMR-1C and TMR-1D may have been formed in an outflow triggered collapse of a string of low-mass cores, or from fragmentation in dense tidal filaments that were generated during an encounter between protostellar disks of TMR-1A and B. In these scenarios, the filaments are either dust material swept-up by the outflow, or tidal arms formed from interacting proto-stellar disks.

- By analyzing spectra extracted at several locations along the filament that "points" towards TMR-1C, we have found evidence of strong molecular hydrogen emission caused by shock excitation. The physical nature of the shocks is described well by slightly curved C-type bow shock models.

- The extinction towards the filament probably increase from the filament's origin, close to the source TMR-1AB, along the filament in the direction of TMR-1C. This has been inferred from extinction values derived from the Q-branch of molecular hydrogen at $2.4-2.5 \mu \mathrm{m}$ and implies that the filament structure is probably receding into the denser parts of the molecular cloud. TMR-1C itself, however, may be sitting slighty in front of the filament's tip, because there are no indications that the extinction along the line of sight to TMR$1 \mathrm{C}$ is higher than towards TMR-1AB. We suggest that the filament is most clearly interpreted in terms of pre-existing dense material being hit by the current outflow, leading to shock emission. An additional component of the filament is continuum emission from scattered light. 
Our results provide new arguments in favour of TMR-1C being a young low-mass object associated with the TMR-1 protostellar system rather than being an extincted background star. Moreover, we have argued that the faint object TMR-1D may also be a component of the TMR-1 system. Unambiguous proof, however, still needs to be found. Confirmation of an edge-on circumstellar disk surrounding TMR-1C would be detection of an infrared excess at $\lambda>10 \mu \mathrm{m}$ and polarized scattered light emission from a disk and/or an envelope. The expected flux from a disk at around $20 \mu \mathrm{m}$ is at a level that is detectable with currently available mid-IR instrumentation on 8-10 m class telescopes, and follow-up observations are critically required. An associated bipolar scattered light structure, however, is probably far more difficult to observe, since the potential size of the disk (and probably also the envelope) is small and is estimated to be $<0.1^{\prime \prime}$, based on the point-source morphology of all HST and ISAAC images. The nature of TMR-1D is currently undetermined and follow-up observations are also needed for this object to fully clarify its nature.

There are currently very few proto-stellar systems known to harbor very low-luminosity wide companions in their immediate surroundings. These systems are particularly important for probing very low-mass star formation models, and TMR-1 is potentially one of those prime-target proto-stellar systems.

Acknowledgements. This work has been carried out based on observations obtained at ESO's LaSilla/Paranal Observatory. The authors thank F. Comeron and F. Bertoldi for valuable suggestions and discussions during early stages of this study. We also thank the referee, J.M. Alcalá, for his detailed and prompt referee report which improved the presentation of this paper. This publication also makes use of data products from the Two Micron All Sky Survey, which is a joint project of the University of Massachusetts and the Infrared Processing and Analysis Center/California Institute of Technology, funded by the National Aeronautics and Space Administration and the National Science Foundation.

\section{References}

Allard, F., Hauschildt, P. H., Alexander, D. R., Tamanai, A., \& Schweitzer, A. 2001, ApJ, 556, 357

Bally, J., \& Reipurth, B. 2001, ApJ, 546, 299

Baraffe, I., Chabrier, G., Barman, T. S., Allard, F., \& Hauschildt, P. H. 2003, A\&A, 402, 701

Brandner, W., Sheppard, S., Zinnecker, H., et al. 2000, A\&A, 364, L13

Burrows, A., Hubbard, W. B., Lunine, J. I., \& Liebert, J. 2001, Rev. Mod. Phys., 73,719

Chabrier, G., Baraffe, I., Allard, F., \& Hauschildt, P. H. 2000, ApJ, 542, 464

Cuby, J. G., Saracco, P., Moorwood, A. F. M., et al. 1999, A\&A, 349, L41

Cuby, J. G., Barucci, A., de Bergh, C., et al. 2000, Proc. SPIE, 4005, 212

Davis, C. J., Dent, W. R. F., Matthews, H. E., Aspin, C., \& Lightfoot, J. F. 1994, MNRAS, 266, 933
Devillard, N. 1997, The eclipse software, The ESO Messenger, 87 Eislöffel, J., Smith, M. D., \& Davis, C. J. 2000, A\&A, 359, 1147

Eislöffel, J., Froebrich, D., Stanke, T., \& McCaughrean, M. J. 2003, ApJ, 595, 259

Furlan, E., McClure, M., Calvet, N., Hartmann, L., et al. 2008, ApJS, 176, 184

Gueth, F., \& Guilloteau, S. 1999, A\&A, 343, 571

Hartmann, L., Calvet, N., Allen, L., Chen, H., \& Jayawardhana, R. 1999, AJ, 118,1784

Hauschildt, P. H., Allard, F., \& Baron, E. 1999, ApJ, 512, 377

Hogerheijde, M. R., van Dishoeck, E. F., Blake, G. A., \& van Langevelde, H. J. 1998, ApJ, 502, 315

Itoh, Y., Tamura, M., \& Nakajima, T. 1999, AJ, 117, 1471

Kenyon, S. J., Calvet, N., \& Hartmann, L. 1993, ApJ, 414, 67

Li, J. Z., Smith, M. D., Gredel, R., Davis, C. J., \& Rector, T. A. 2008, ApJ, 679, L101

Lin, D. N. C., Laughlin, G., Bodenheimer, P., \& Rozyczka, M. 1998, Science, 281,2025

Lucas, P. W., Roche, P. F., Allard, F., \& Hauschildt, P. H. 2001, MNRAS, 326, 695

Lucas, P. W., Weights, D. J., Roche, P. F., \& Riddick, F. C. 2006, MNRAS, 373, L60

Luhman, K. L., Adame, L., D’Alessio, P., et al. 2007, ApJ, 666, 1219

Motte, F., \& André, P. 2001, A\&A, 365, 440

Natta, A., Testi, L., Comerón, F., et al. 2002, A\&A, 393, 597

O'Connell, B., Smith, M. D., Froebrich, D., Davis, C. J., \& Eislöffel, J. 2005, A\&A, 431, 223

Ohashi, N., Hayashi, M., Kawabe, R., \& Ishiguro, M. 1996, ApJ, 466, 317

Padoan, P., \& Nordlund, A. 2004, ApJ, 617, 559

Petr-Gotzens, M. G., Cuby, J. G., Sterzik, M. F., Schilke, P., \& Walsh, A. 2002, in The Origins of Stars and Planets: The VLT View, ed. J. Alves, \& M. J. McCaughrean, Proc. ESO Workshop held in Garching, Germany, 24-27 April 2001, 391

Pickles, A. J. 1998, PASP, 110, 863

Prato, L., Lockhart, K. E., Johns-Krull, C. M., \& Rayner, J. T. 2009, AJ, 137, 3931

Robitaille, T. P., Whitney, B. A., Indebetouw, R., \& Wood, K. 2007, ApJS, 169, 328

Sandell, G., \& Knee, L. B. G. 2001, ApJ, 546, L49

Scholz, A., Jayawardhana, R., Wood, K., et al. 2008, ApJ, 681, L29

Shen, S., \& Wadsley, J. 2006, ApJ, 651, L145

Shull, J. M., \& Beckwith, S. 1982, ARA\&A, 20, 163

Smith, M. D. 2000, IrAJ, 27, 37

Smith, M. D., \& Brand, P. W. J. L. 1990, MNRAS, 243, 498

Smith, M. D., Davis, C. J., \& Lioure, A. 1997, A\&A, 327, 1206

Stamatellos, D., \& Whitworth, A. P. 2009, MNRAS, 392, 413

Terebey, S., Beichmann, C. A., Gautier, T. N., \& Hester, J. J. 1990, ApJ, 362, L63

Terebey, S., van Buren, D., Padgett, D. L., Hancock, T., \& Brundage, M. 1998, ApJ, 507, L71

Terebey, S., van Buren, D., Matthews, K., \& Padgett, D. L. 2000, AJ, 119, 2341

Torres, R. M., Loinard, L., Mioduszewski, A. J., \& Rodríguez, L. F. 2007, ApJ, 671,1813

Wilking, B. A., Greene, T. P., \& Meyer, M. R. 1999, AJ, 117, 469

Whitney, B. A., Kenyon, S. J., \& Gómez, M. 1997, ApJ, 485, 703 